Review Article

\title{
Kochiae Fructus, the Fruit of Common Potherb Kochia scoparia (L.) Schrad: A Review on Phytochemistry, Pharmacology, Toxicology, Quality Control, and Pharmacokinetics
}

\author{
Wei Zou, ${ }^{1}$ Zhong Tang, ${ }^{2}$ Yao Long, ${ }^{1}$ Zuoqi Xiao, ${ }^{1}$ Bo Ouyang $\mathbb{D},{ }^{1}$ and Menghua Liu $\mathbb{D D}^{3}$ \\ ${ }^{1}$ NHC Key Laboratory of Birth Defects Research, Prevention and Treatment, Hunan Provincial Maternal and Child Health \\ Care Hospital, Changsha 410008, China \\ ${ }^{2}$ Breast Disease Department, The First Hospital of Hunan University of Chinese Medicine, Changsha 410007, China \\ ${ }^{3}$ Guangdong Provincial Key Laboratory of New Drug Screening, School of Pharmaceutical Sciences, Southern Medical University, \\ Guangzhou 510515, China
}

Correspondence should be addressed to Bo Ouyang; 1050495772@qq.com and Menghua Liu; liumenghua@smu.edu.cn

Received 19 June 2020; Revised 8 December 2020; Accepted 19 January 2021; Published 31 January 2021

Academic Editor: Jiangyong Gu

Copyright ( $) 2021$ Wei Zou et al. This is an open access article distributed under the Creative Commons Attribution License, which permits unrestricted use, distribution, and reproduction in any medium, provided the original work is properly cited.

\begin{abstract}
Kochiae Fructus (KF) is the fruit of an annual potherb Kochia scoparia (Linn.) Schrad and has been traditionally used for the treatment of diseases in the skin, eyes, and urinary tract for thousands of years in China. Recent studies have showed its anti-inflammatory, antifungal, antiallergic, and antipruritogenic effects to clarify the mechanisms of these actions. Meanwhile, its other effects, such as anticancer, hypoglycemic, and hepatoprotective effects, also have been reported. The achievement of these therapeutic effects is contributed by its chemical constituents. A total of 153 compounds have been identified in KF, mainly including triterpenoids, flavonoids, carbohydrates, amino acids, organic acids, and essential oils. Momordin Ic is the representative triterpene glycoside compound, which is used as a phytochemical marker for the quality control of Kochiae Fructus. The research on toxicity is insufficient, and only one article reported that the $\mathrm{LD}_{50} \mathrm{was}$ $7.15 \pm 0.03 \mathrm{~g} / \mathrm{kg}$ for water extract of KF after oral administration in KM mice. In addition, the pharmacokinetic study was carried out on momordin Ic with linear pharmacokinetic characteristics. Above all, this review provides comprehensive information about Kochiae Fructus and may provide the theoretic foundation of its clinical application and further development.
\end{abstract}

\section{Introduction}

Kochia scoparia (Linn.) Schrad (shown in Figure 1(a)), also called Bassia scoparia (L.) A.J. Scott, is a large annual potherb in the family Chenopodiaceae widely distributed in Europe and Asia and naturalized in Africa, Australia, and North and South America [1]. Kochia Fructus (KF, shown in Figure 1(b)) is the fruit of Kochia scoparia, which is a spheroidal pentagram with a diameter of 1 to $3 \mathrm{~mm}$ [2]. It was first recorded in "Shennong Ben Cao Jing" as a "top grade" medicinal material. Up to now, KF has been used in traditional Chinese and Japanese medicine more than 2000 years for the treatment of diseases of the skin, eyes, and urinary tract [3]. With the deepening and development of pharmacology research, it has attracted attention particularly because of its antibacterial, anti-inflammatory, antiallergic, antigastric mucosal damage, hypoglycemic, and immunity enhancing effects [4]. Recently, researchers demonstrated that KF mainly contains terpenoids, flavonoids, essential oils, trace elements, and other ingredients. Although there were many researches on the chemical constituents, pharmacological activities of $\mathrm{KF}$, a systematic and updated review is unavailable. Therefore, the aim of this review is to extensively summarize the phytochemistry, pharmacology, quality control, toxicology, and pharmacokinetics of KF, as well as providing novel insights for clinical uses and further researches. 


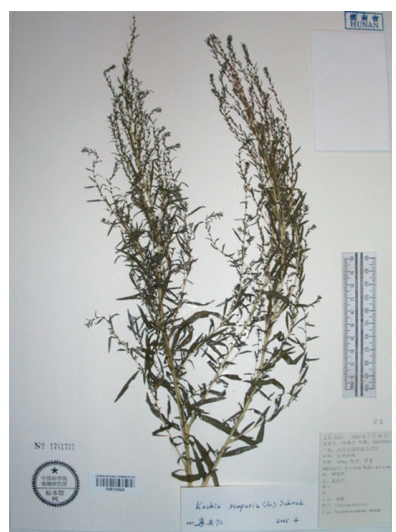

(a)

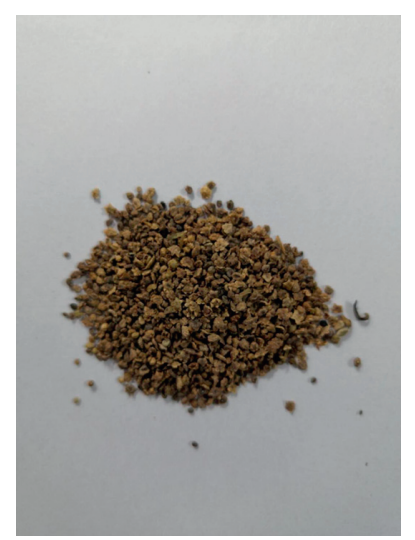

(b)
Figure 1: (a) The potherb of Kochia scoparia (L.) Schrad (http:// www.cvh.ac.cn/spms/detail.php?id=f27a89aa) and (b) its fruit Kochiae Fructus.

\section{Phytochemistry}

With the advancement of analysis technologies such as liquid chromatograph-mass spectrometer (LC-MS), nuclear magnetic resonance-mass spectrometer (NMR-MS), and gas chromatography-mass spectrometer (GC-MS), identification of various components in traditional Chinese medicine has been simplified. To date, 153 compounds within KF, including 25 triterpenoids, 13 flavonoids, 22 carbohydrates (primarily mono- and disaccharides), 21 amino acids, 9 organic acid, 49 essential oils, and 14 heterocyclics, have been identified (Table 1 and Figure 2). Most of investigations indicated that triterpenoids are the main active ingredient within KF. They were characterized with tetracyclic or pentacyclic rings by the polymerization of isoprene. Among them, momordin Ic is a representative triterpenoid saponin with anti-inflammatory effect [5]. Flavonoids were another major component within KF [12]. Most of them are derivatives of flavonol aglycones including quercetin and isorhamnetin. The carbohydrates of flavonoid glycosides are glucopyranose, rhamnose, and galactose. Besides, other flavonoids such as 5,7,4'-trihydroxy-6,3'-dimethoxyflavone and 5,7,4'-dihydroxy-6-methoxyflavone were characterized by LC-MS [6]. KF contains many kinds of amino acid, and current research suggests that certain functional amino acids can play a pharmacological role through the gut-microbiome-immune axis [17]. The essential oil within KF is high fatty acid ester. Yang et al. used a supercritical $\mathrm{CO}_{2}$ extraction method combined with a gas chromatography-mass spectrometer (GC-MS) method to qualify these essential oil components [14]. Eighteen compounds were isolated and identified, most of which were fatty acid esters and aromatic compounds. The level of the higher fatty acid ester is high, and the relative amount of 9,12-octadecadienoic acid is the highest in oil, followed by 9 -octadecenoic acid. Wen et al. used the GC-MS method to qualitatively analyze the essential oil within KF [15]. Compared with the standard mass spectrum, 36 components were identified. Among them, the relative level of high fatty acid esters is the highest, and the amount of terpenoids is small.

\section{Pharmacology}

Traditionally, according to records of "Shennong Ben Cao Jing," KF was used with the therapeutic effects of diuresis and benefiting pneuma. Compendium of Materia Medica described that the KF could be used in the treatment of red eyes, hemntodiarrhoea, pregnancy combined with gonorrhea, and urinary stoppage [18]. Many other books also depicted the traditional use of KF, which are summarized in Table 2. Modern investigations have proved that KF has antiinflammatory, hypoglycemic, anticancer, antifungal, antipruritogenic, and antinociceptive effects, as well as antiallergic, antiedema, and hepatoprotective activities. We have enlisted an overview of the pharmacological studies in the following sections (Table 3).

3.1. Anti-Inflammatory Effect. Pharmacological studies showed that anti-inflammatory is a very significant pharmacological activity of KF. In six different animal models (the ddY mice in an acetic acid-induced vascular permeability, the ddY mice in a carrageenin-induced edema, the ddY mice in a compound 48/80-induced edema, the ddY mice in a chemical mediator-induced edema, the ddY mice in an arachidonic acid-induced edema, a picryl chlorideinduced ear inflammatory model in ICR mice), the $70 \%$ alcohol extract of KF has been proved with obvious inhibition effect on the development of inflammation $[19,20]$. The methanol extract of KF was used as a candidate drug for the treatment of inflammatory skin diseases due to its eutherapeutic effect on 1-fluoro-2,4-dinitrofluorobenzeneinduced contact dermatitis mice model. The mechanism might be involved in inhibiting the skewing reaction of $\mathrm{T}$ helper cell type 1 [22]. The total flavonoids of the KF have shown an anti-inflammatory effect on the dinitrochlorobenzene-induced allergic contact dermatitis rats, and the most likely mechanism of this action involves regulating pERK1/2/TLR4-NF- $\kappa$ B pathway activation [42]. The antiinflammatory effect of KF was coincident with its traditional use for inflammations in vagina and skin.

Three triterpenoid saponins, namely, 20-hydroxyecdysone, momordin Ic, and oleanolic acid from KF have also been investigated on LPS-stimulated murine macrophage RAW 264.7 cell line. 20-Hydroxyecdysone performed significant inhibitory action on prostaglandin $\mathrm{E}_{2}\left(\mathrm{PGE}_{2}\right)$ generation at the dose of $12.5 \mu \mathrm{M}$, while momordin Ic and oleanolic acid showed the anti-inflammatory effect at the dose of $6.25 \mu \mathrm{M}$. In addition, momordin Ic significantly reduced productions of tumor necrosis factor-alpha (TNF$\alpha$ ) and interleukin-6 (IL-6) at the concentration of $12.5 \mu \mathrm{M}$ [5].

3.2. Hypoglycemic Effect. KF has shown a potential hypoglycemic effect. Dai et al. illustrated that $n$-butanol fraction of KF could markedly inhibit gastric emptying in normal mice and could more potently inhibit gastric emptying in hyperglycemic and hypoglycemic mice at the dose of $25 \mathrm{mg} /$ $\mathrm{kg}$. The hypoglycemic mechanism is probably related to transportation and transformation of sugar in the digestive 
Table 1: Chemical compounds identified in Kochiae Fructus.

\begin{tabular}{|c|c|c|c|}
\hline No. & Compounds & Formula & Ref. \\
\hline \multicolumn{4}{|c|}{ Triterpenoids } \\
\hline 1 & 20-Hydroxyecdysone & $\mathrm{C}_{27} \mathrm{H}_{44} \mathrm{O}_{7}$ & {$[5]$} \\
\hline 2 & $\beta$-Sitosterol & $\mathrm{C}_{29} \mathrm{H}_{50} \mathrm{O}$ & {$[6]$} \\
\hline 3 & Momordin Ic & $\mathrm{C}_{41} \mathrm{H}_{64} \mathrm{O}_{13}$ & [7] \\
\hline 4 & Oleanic-acid-3-O- $\beta$-D-xylopyranosly $(1 \longrightarrow 3)-\beta$-D-glucopyranoside & $\mathrm{C}_{41} \mathrm{H}_{65} \mathrm{O}_{12}$ & [7] \\
\hline 5 & Momordin I & $\mathrm{C}_{41} \mathrm{H}_{64} \mathrm{O}_{13}$ & [7] \\
\hline 6 & Oleanolic acid & $\mathrm{C}_{30} \mathrm{H}_{48} \mathrm{O}_{3}$ & [7] \\
\hline 7 & Oleanolic acid 3-O-glucuronide & $\mathrm{C}_{36} \mathrm{H}_{56} \mathrm{O}_{9}$ & [7] \\
\hline 8 & Oleanolic acid 3-O- $\beta$-D-glucopyranoside & $\mathrm{C}_{36} \mathrm{H}_{56} \mathrm{O}_{8}$ & [7] \\
\hline 9 & 28-O-Deglucosyl-chikusetsusaponin V & $\mathrm{C}_{42} \mathrm{H}_{66} \mathrm{O}_{14}$ & [7] \\
\hline 10 & 2'-O-Glucopyranosyl-momordin Ic & $\mathrm{C}_{48} \mathrm{H}_{76} \mathrm{O}_{19}$ & [7] \\
\hline 11 & Chikusetsusaponin V & $\mathrm{C}_{48} \mathrm{H}_{76} \mathrm{O}_{19}$ & [7] \\
\hline 12 & Momordin IIc & $\mathrm{C}_{47} \mathrm{H}_{74} \mathrm{O}_{18}$ & {$[7]$} \\
\hline 13 & Kochianoside I & $\mathrm{C}_{36} \mathrm{H}_{56} \mathrm{O}_{10}$ & {$[8]$} \\
\hline 14 & Kochianoside II & $\mathrm{C}_{47} \mathrm{H}_{74} \mathrm{O}_{18}$ & {$[8]$} \\
\hline 15 & Kochianoside III & $\mathrm{C}_{41} \mathrm{H}_{64} \mathrm{O}_{14}$ & {$[8]$} \\
\hline 16 & Kochianoside IV & $\mathrm{C}_{41} \mathrm{H}_{64} \mathrm{O}_{13}$ & {$[8]$} \\
\hline 17 & Daucosterol & $\mathrm{C}_{35} \mathrm{H}_{60} \mathrm{O}_{6}$ & {$[6]$} \\
\hline 18 & Oleanolic acid 3-O- $\beta$-D-xylopyranosyl $(1 \longrightarrow 3)-\beta$-D-glucopyranosiduronic acid 6-methyl ester & $\mathrm{C}_{42} \mathrm{H}_{66} \mathrm{O}_{13}$ & {$[6]$} \\
\hline 19 & 3-O- $\beta$-D-Glucuronopyranosy-28-O- $\beta$-D-glucuronopyranosyl-oleanic-acid & $\mathrm{C}_{42} \mathrm{H}_{66} \mathrm{O}_{14}$ & [6] \\
\hline 20 & Oleanolic acid 3- $\beta$-D-glucopyranosiduronic acid 6-methyl ester & $\mathrm{C}_{37} \mathrm{H}_{58} \mathrm{O}_{9}$ & [9] \\
\hline 21 & Stigmasterol-3-O- $\beta$-D-glucopyranoside & $\mathrm{C}_{35} \mathrm{H}_{58} \mathrm{O}_{6}$ & [8] \\
\hline 22 & Oleanic-acid-3-O-[ $\beta$-D-glucopyranosyl $(1 \longrightarrow 2)-\beta$-D-xylopyranosly $(1 \longrightarrow 3)]-\beta$-D-glucopyranosiduronic acid & $\mathrm{C}_{47} \mathrm{H}_{74} \mathrm{O}_{18}$ & [10] \\
\hline 23 & $6^{\prime}$-Methyl ester of momordin Ic & $\mathrm{C}_{42} \mathrm{H}_{66} \mathrm{O}_{13}$ & [11] \\
\hline 24 & $2^{\prime}-O-\beta$-D-Glucopyranosyl momordin Ic & $\mathrm{C}_{47} \mathrm{H}_{74} \mathrm{O}_{18}$ & [11] \\
\hline 25 & $2^{\prime}-O-\beta$-D-Glucopyranosyl momordin IIc & $\mathrm{C}_{53} \mathrm{H}_{84} \mathrm{O}_{23}$ & [11] \\
\hline \multicolumn{4}{|c|}{ Flavonoids } \\
\hline 26 & Quercetin 3-O- $\beta$-D-apiofuranosyl-(1 $\longrightarrow 2)-\beta$-D-galactopyranosyl-7-O- $\beta$-D-glucopyranoside & $\mathrm{C}_{32} \mathrm{H}_{38} \mathrm{O}_{21}$ & [12] \\
\hline 27 & Quercetin 3-O- $\alpha$-L-rhamnopyranosyl-(1 $\longrightarrow 6)-\beta$-D-galactopyranosyl-7-O- $\beta$-D-sophoroside & $\mathrm{C}_{39} \mathrm{H}_{50} \mathrm{O}_{26}$ & [12] \\
\hline 28 & Quercetin 7-O- $\beta$-D-glucopyranoside & $\mathrm{C}_{21} \mathrm{H}_{20} \mathrm{O}_{12}$ & [12] \\
\hline 29 & Quercetin 3-O- $\beta$-D-sophoroside & $\mathrm{C}_{27} \mathrm{H}_{30} \mathrm{O}_{17}$ & [12] \\
\hline 30 & Quercetin 3-O- $\beta$-D-galactopyranosyl-7-O- $\beta$-D-glucopyranoside & $\mathrm{C}_{27} \mathrm{H}_{30} \mathrm{O}_{17}$ & [12] \\
\hline 31 & Quercetin $3-O-\beta$-D-apiofuranosyl-(1 $\longrightarrow 2)$ - $\beta$-D-galactopyranoside & $\mathrm{C}_{26} \mathrm{H}_{28} \mathrm{O}_{16}$ & [12] \\
\hline 32 & Isorhamnetin & $\mathrm{C}_{16} \mathrm{H}_{12} \mathrm{O}_{7}$ & {$[6]$} \\
\hline 33 & Quercetin & $\mathrm{C}_{15} \mathrm{H}_{10} \mathrm{O}_{7}$ & {$[6]$} \\
\hline 34 & Rutin & $\mathrm{C}_{27} \mathrm{H}_{30} \mathrm{O}_{16}$ & [6] \\
\hline 35 & Isorhamnetin-3-O-glucoside & $\mathrm{C}_{22} \mathrm{H}_{22} \mathrm{O}_{12}$ & {$[6]$} \\
\hline 36 & $5,7,4^{\prime}$-Trihydroxy- $6,3^{\prime}$-dimethoxyflavone & $\mathrm{C}_{17} \mathrm{H}_{14} \mathrm{O}_{7}$ & [6] \\
\hline 37 & 5,7,4'-Dihydroxy-6-methoxyflavone & $\mathrm{C}_{16} \mathrm{H}_{12} \mathrm{O}_{6}$ & {$[6]$} \\
\hline 38 & Hyperoside & $\mathrm{C}_{21} \mathrm{H}_{20} \mathrm{O}_{12}$ & [10] \\
\hline \multicolumn{4}{|c|}{ Carbohydrates } \\
\hline 39 & Sorbitol & $\mathrm{C}_{6} \mathrm{H}_{14} \mathrm{O}_{6}$ & [13] \\
\hline 40 & Glucose & $\mathrm{C}_{6} \mathrm{H}_{12} \mathrm{O}_{6}$ & [13] \\
\hline 41 & Galactopyranose & $\mathrm{C}_{6} \mathrm{H}_{12} \mathrm{O}_{6}$ & [13] \\
\hline 42 & Fructose & $\mathrm{C}_{6} \mathrm{H}_{12} \mathrm{O}_{6}$ & [13] \\
\hline 43 & Inositol-scyllo & & [13] \\
\hline 44 & Trehalose & $\mathrm{C}_{12} \mathrm{H}_{22} \mathrm{O}_{11}$ & [13] \\
\hline 45 & Sucrose & $\mathrm{C}_{12} \mathrm{H}_{22} \mathrm{O}_{11}$ & [13] \\
\hline 46 & $N$-Acetyl glucosamine & $\mathrm{C}_{8} \mathrm{H}_{15} \mathrm{NO}_{6}$ & [13] \\
\hline 47 & Mannitol & $\mathrm{C}_{6} \mathrm{H}_{14} \mathrm{O}_{6}$ & [13] \\
\hline 48 & Mannose & $\mathrm{C}_{6} \mathrm{H}_{12} \mathrm{O}_{6}$ & [13] \\
\hline 49 & Glucosamine & $\mathrm{C}_{6} \mathrm{H}_{13} \mathrm{NO}_{5}$ & [13] \\
\hline 50 & Glycerophosphoric acid & $\mathrm{C}_{3} \mathrm{H}_{9} \mathrm{O}_{6} \mathrm{P}$ & [13] \\
\hline 51 & Rhamnose & $\mathrm{C}_{6} \mathrm{H}_{12} \mathrm{O}_{5}$ & [13] \\
\hline 52 & Ribitol & $\mathrm{C}_{5} \mathrm{H}_{12} \mathrm{O}_{5}$ & [13] \\
\hline 53 & Xylulose & $\mathrm{C}_{5} \mathrm{H}_{10} \mathrm{O}_{5}$ & [13] \\
\hline 54 & Erythritol & $\mathrm{C}_{4} \mathrm{H}_{10} \mathrm{O}_{4}$ & [13] \\
\hline 55 & 2-O-Glycerol- $\alpha$-D-galactopyranoside & $\mathrm{C}_{9} \mathrm{H}_{18} \mathrm{O}_{8}$ & [13] \\
\hline 56 & Maltitol & $\mathrm{C}_{12} \mathrm{H}_{24} \mathrm{O}_{11}$ & [13] \\
\hline 57 & Melibiose & $\mathrm{C}_{12} \mathrm{H}_{22} \mathrm{O}_{11}$ & [13] \\
\hline
\end{tabular}


TABle 1: Continued.

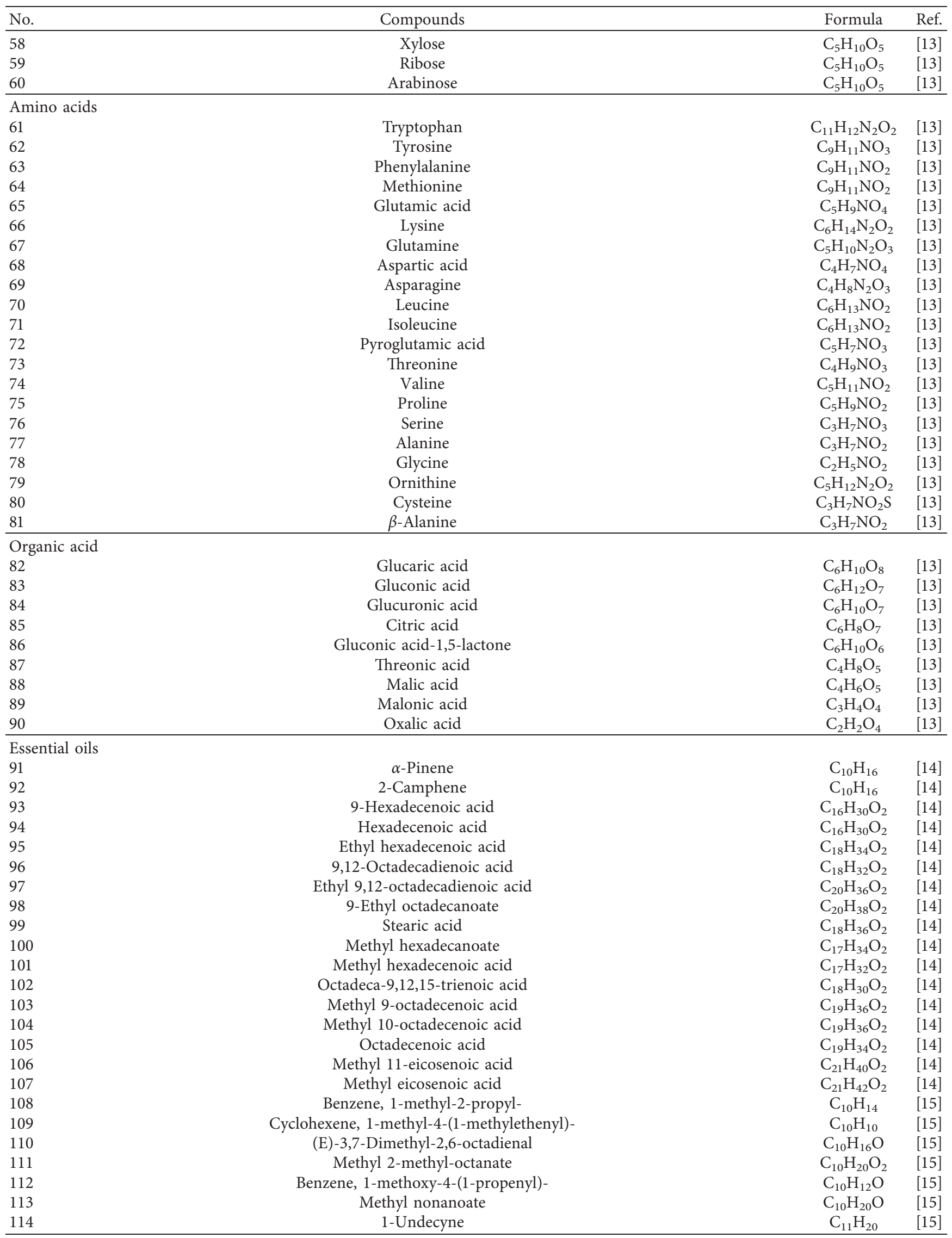


TABLE 1: Continued.

\begin{tabular}{|c|c|c|c|}
\hline No. & Compounds & Formula & Ref. \\
\hline 115 & Ethyl nonanoate & $\mathrm{C}_{11} \mathrm{H}_{22} \mathrm{O}_{2}$ & {$[15]$} \\
\hline 116 & Naphthalene & $\mathrm{C}_{11} \mathrm{H}_{10}$ & [15] \\
\hline 117 & $\operatorname{Bicyclo}(4,3,1)$ decan-10-one & $\mathrm{C}_{10} \mathrm{H}_{16} \mathrm{O}$ & [15] \\
\hline 118 & 5-Ethyl-2-nonanol & $\mathrm{C}_{11} \mathrm{H}_{24} \mathrm{O}$ & {$[15]$} \\
\hline 119 & 4,8-Dimethyl-1-nonanol & $\mathrm{C}_{11} \mathrm{H}_{24} \mathrm{O}$ & [15] \\
\hline 120 & (Z)- $\beta$-Farnesene & $\mathrm{C}_{15} \mathrm{H}_{24}$ & [15] \\
\hline 121 & trans- $\beta$-Farnesene & $\mathrm{C}_{15} \mathrm{H}_{24}$ & [15] \\
\hline 122 & 2,6-Di-T-butyl-1,4-benzoquinone & $\mathrm{C}_{14} \mathrm{H}_{20} \mathrm{O}$ & [15] \\
\hline 123 & $\beta$-Ionone & $\mathrm{C}_{13} \mathrm{H}_{20} \mathrm{O}$ & [15] \\
\hline 124 & Naphthalene, 1,2,3,5,6,7,8a-octahydro-1,8a-demethyl-7-(1-methyethyl)-[1R-(1 $\alpha, 7 \beta, 8 \mathrm{a} \alpha)]$ - & $\mathrm{C}_{15} \mathrm{H}_{24}$ & [15] \\
\hline 125 & $\beta$-Chamigrene & $\mathrm{C}_{15} \mathrm{H}_{24}$ & [15] \\
\hline 126 & Ethyl dodecanoate & $\mathrm{C}_{14} \mathrm{H}_{28} \mathrm{O}_{2}$ & [15] \\
\hline 127 & Hexadecane & $\mathrm{C}_{16} \mathrm{H}_{34}$ & [15] \\
\hline 128 & Methyl tetradecanoate & $\mathrm{C}_{15} \mathrm{H}_{30} \mathrm{O}_{2}$ & [15] \\
\hline 129 & Benzene, 1,1'-(1,2-ethynediyl)bis- & $\mathrm{C}_{14} \mathrm{H}_{10}$ & [15] \\
\hline 130 & Octadecane & $\mathrm{C}_{18} \mathrm{H}_{38}$ & [15] \\
\hline 131 & 2-Pentadecanone, 6,10,14-trimethyl- & $\mathrm{C}_{18} \mathrm{H}_{36} \mathrm{O}$ & [15] \\
\hline 132 & Ethyl pentadecanoate & $\mathrm{C}_{17} \mathrm{H}_{34} \mathrm{O}_{2}$ & {$[15]$} \\
\hline 133 & Ethyl hexadecanoate & $\mathrm{C}_{18} \mathrm{H}_{36} \mathrm{O}_{2}$ & {$[15]$} \\
\hline 134 & Eicosane & $\mathrm{C}_{20} \mathrm{H}_{42}$ & [15] \\
\hline 135 & Methyl octadecadienoate & $\mathrm{C}_{19} \mathrm{H}_{34} \mathrm{O}_{2}$ & [15] \\
\hline 136 & Heneicosane & $\mathrm{C}_{21} \mathrm{H}_{44}$ & [15] \\
\hline 137 & Ethyl-octadecadienoate & $\mathrm{C}_{20} \mathrm{H}_{36} \mathrm{O}_{2}$ & [15] \\
\hline 138 & Ethyl octadecanoate & $\mathrm{C}_{20} \mathrm{H}_{40} \mathrm{O}_{2}$ & [15] \\
\hline 139 & Docosane & $\mathrm{C}_{22} \mathrm{H}_{46}$ & [15] \\
\hline \multicolumn{4}{|c|}{ Heterocyclics } \\
\hline 140 & Oleamide & $\mathrm{C}_{18} \mathrm{H}_{35} \mathrm{NO}$ & [13] \\
\hline 141 & Ethanolamine & $\mathrm{C}_{2} \mathrm{H}_{7} \mathrm{NO}$ & [13] \\
\hline 142 & Uridine & $\mathrm{C}_{9} \mathrm{H}_{12} \mathrm{~N}_{2} \mathrm{O}_{6}$ & [13] \\
\hline 143 & Uric acid & $\mathrm{C}_{5} \mathrm{H}_{4} \mathrm{~N}_{4} \mathrm{O}_{3}$ & [13] \\
\hline 144 & Guanine & $\mathrm{C}_{5} \mathrm{H}_{5} \mathrm{~N}_{5} \mathrm{O}$ & [13] \\
\hline 145 & Adenine & $\mathrm{C}_{5} \mathrm{H}_{5} \mathrm{~N}_{5}$ & {$[13]$} \\
\hline 146 & Spermidine & $\mathrm{C}_{7} \mathrm{H}_{19} \mathrm{~N}_{3}$ & {$[13]$} \\
\hline 147 & Putrescine & $\mathrm{C}_{4} \mathrm{H}_{12} \mathrm{~N}_{2}$ & {$[13]$} \\
\hline 148 & Ethylene glycol & $\mathrm{C}_{2} \mathrm{H}_{6} \mathrm{O}_{2}$ & [13] \\
\hline 149 & Urea & $\mathrm{CH}_{4} \mathrm{~N}_{2} \mathrm{O}$ & [13] \\
\hline 150 & Allantoin & $\mathrm{C}_{4} \mathrm{H}_{6} \mathrm{~N}_{4} \mathrm{O}_{3}$ & [13] \\
\hline 151 & Thymine & $\mathrm{C}_{5} \mathrm{H}_{6} \mathrm{~N}_{2} \mathrm{O}_{2}$ & {$[13]$} \\
\hline 152 & 2-(4-Hydroxy-3-methoxyphenyl)-ethanol & $\mathrm{C}_{9} \mathrm{H}_{12} \mathrm{O}_{3}$ & {$[13]$} \\
\hline 153 & Dopamine & $\mathrm{C}_{8} \mathrm{H}_{11} \mathrm{NO}_{2}$ & {$[16]$} \\
\hline
\end{tabular}

tract and absorption of glucose via the membrane of the small intestine [23]. Subsequently, a research on the function of small intestine was implemented and it found that the $n$ butanol fraction with a dose of $50 \mathrm{mg} / \mathrm{kg}$ could improve the propulsive function of small intestine, and the mechanism of this action probably involves cholinergic nerve and nitric oxide [24].

Matsuda et al. found that momordin Ic inhibited gastric emptying in rats and inhibited glucose uptake in the small intestine in vitro, which contributed to the hypoglycemic action of momordin Ic [25]. Further study showed that momordin Ic inhibits gastric emptying in normal mice, hyperglycemic (including diabetic) and hypoglycemic mice, nonnutrient meal-loaded mice, and nutrient meal-loaded mice [3]. When gastric emptying is slow, the postprandial absorption of food will prolong. Hence, the inhibition of gastric emptying induced by momordin Ic may be useful for the prevention and treatment of diabetes and the morbid obesity with accelerated gastric emptying.

\subsection{Anticancer Effect}

3.3.1. Antiliver Cancer Effect. Momordin Ic was the main triterpenoid saponins within KF and has showed an antiliver cancer effect. Wang et al. have carried out a series of researches and found that HepG2 cells were sensitive to the cytotoxic effect of momordin Ic. Momordin Ic could induce apoptosis through oxidative stress-regulated mitochondrial dysfunction involving MAPK and PI3K-mediated iNOS and HO-1 pathways [26]. Based on these results, Wang et al. investigated the MAPK and PI3K pathways and their downstream proteins, such as PPARg and COX-2. Then, they provided the evidence that momordin Ic-induced HepG2 cell apoptosis was associated with PI3K and MAPK pathway-mediated PPARg activation [28]. In addition, $\mathrm{Mi}$ 

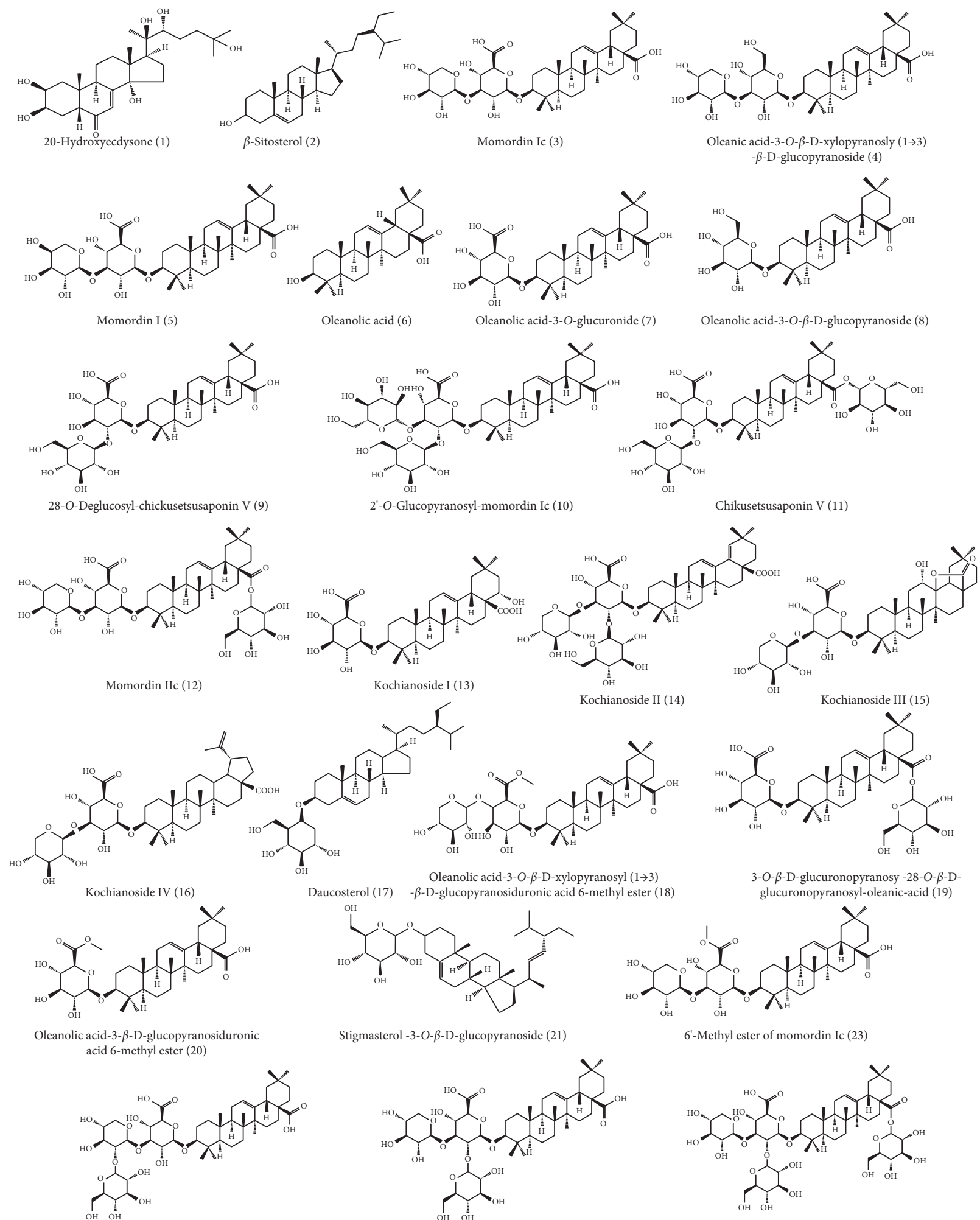

Oleanic-acid-3-O-[ $\beta$-D-glucopyranosyl $(1 \rightarrow 2)-\beta$-D xylopyranosly $(1 \rightarrow 3)]-\beta$-D-glucopyranosiduronic acid (22)

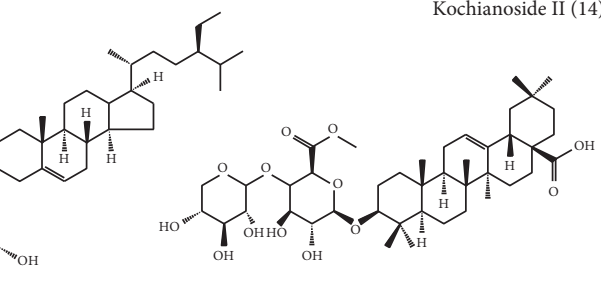

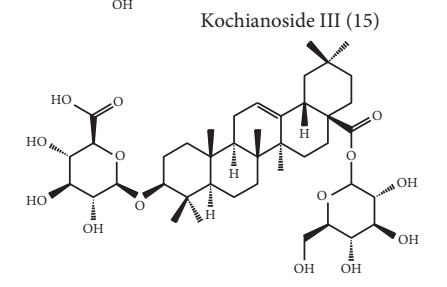

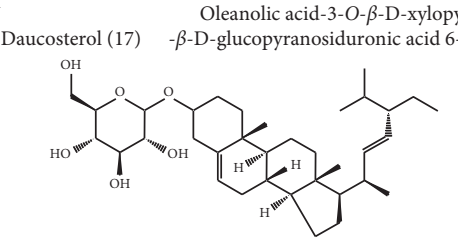

Stigmasterol -3-O- $\beta$-D-glucopyranoside (21)

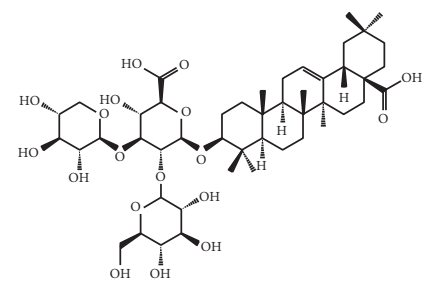

2'-O- $\beta$-D-Glucopyranosyl momordin Ic (24)

(a)
3-O- $\beta$-D-glucuronopyranosy -28-O- $\beta$-Dglucuronopyranosyl-oleanic-acid (19)

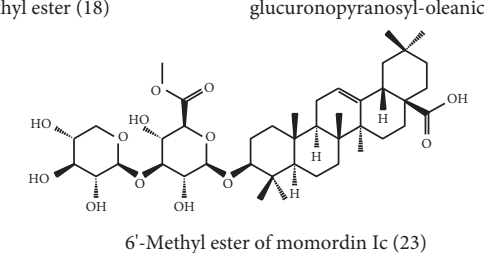

6'-Methyl ester of momordin Ic (23)

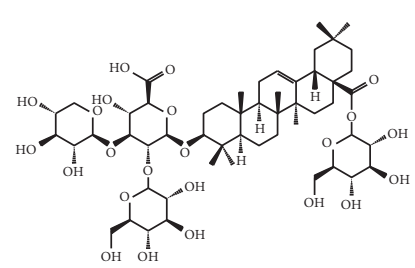

2'-O- $\beta$-D-Glucopyranosyl momordin IIc (25)

Figure 2: Continued. 


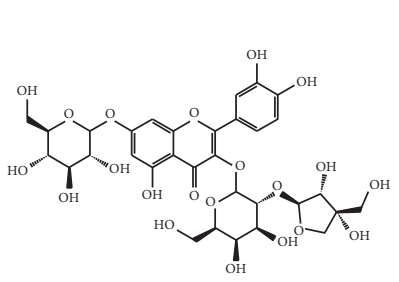

Quercetin-3-O- $\beta$-D-apiofuranosyl$(1 \rightarrow 2)-\beta$-D-galactopyranosyl-7-O$\beta$-D-glucopyranoside (26)

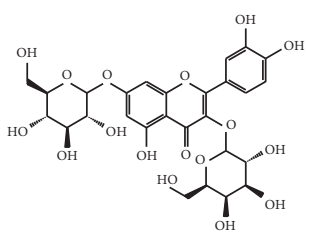

Quercetin-3-O- $\beta$-D-galactopyranosyl7-O- $\beta$-D-glucopyranoside (30)

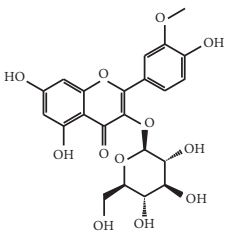

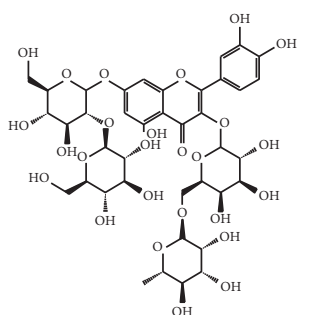

Quercetin-3-O- $\alpha$-L-rhamnopyranosyl- $(1 \rightarrow 6)$ - $\beta$-D-galactopyranosyl-7-O- $\beta$-D-sophoroside (27)

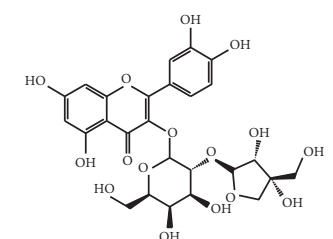

Quercetin-3-O- $\beta$-D-apiofuranosyl$(1 \rightarrow 2)-\beta$-D-galactopyranoside (31)<smiles>COc1cc(C2Oc3cc(O)cc(O)c3C(=O)C2O)ccc1O</smiles>

Isorhamnetin (32)

Quercetin (33)

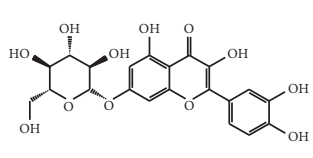

Quercetin-7-O- $\beta$-D-
glucopyranoside (28)

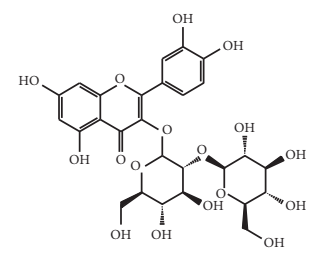

Quercetin-3-O- $\beta$-Dsophoroside (29)

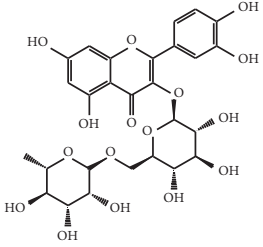

Rutin (34)

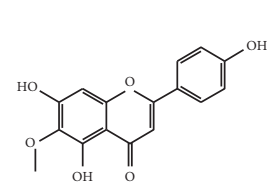

Isorhamnetin-3-O-glucoside (35) 5, 7, 4'-Dihydroxy-6-methoxyflavon (36)

Sorbitol (39)

N-Acetyl glucosamine (46)<smiles>CC(=O)NC[C@@H]1O[C@H](CO)[C@@H](O)C(O)C1NC(C)=O</smiles>

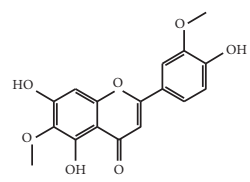

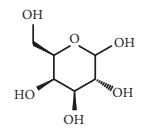

Galactopyranose (41)

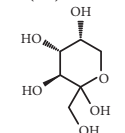

Fructose (42)

5, 7, 4'-Trihydroxy-6, 3'-dimethoxyflavon (37)

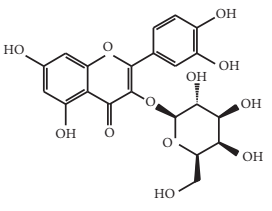

Hyperoside (38)<smiles>N[C@H]1C(O)OC(CO)[C@H](O)[C@H]1O</smiles>

Glucosamine (49)

$$
\mathrm{OH} \quad \mathrm{OH}
$$<smiles>C[C@H]1OC(O)[C@H](O)[C@H](O)[C@H]1O</smiles><smiles>O=C(CO)[C@@H](O)[C@@H](O)CO</smiles>

Rhamnose (51)<smiles>OC1OC[C@@H](O)[C@H](O)C1O</smiles>

Xylose (58)<smiles>CSCC[C@H](N)C(=O)O</smiles>

Methionine (64)<smiles>OC1OC[C@@H](O)[C@@H](O)[C@@H]1O</smiles>

Ribose (59)

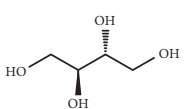

Erythritol (54)<smiles>CC1COC(O)[C@H](O)[C@H]1O</smiles>

Arabinose (60)<smiles>CCC(CC)OC1O[C@H](O)[C@@H](O)C(O)C1O</smiles>

$$
\text { 2-O-Glycerol- } \alpha \text {-D- }
$$
galactopyranoside (55)

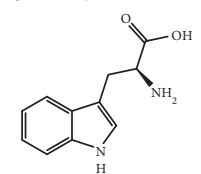

Tryptophan (61)

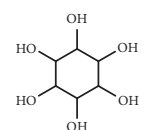

Inositol-scyllo (43)
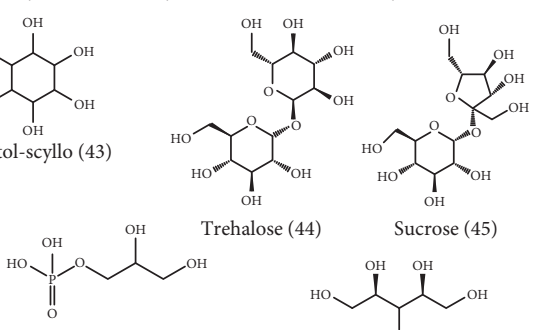

Glycerophosphoric acid (50)<smiles>OCC(O)C(O)C(O)CO</smiles><smiles>C[C@H]1OC(COC2OC(CO)[C@@H](O)C(O)C2O)C(O)C(O)C1O</smiles><smiles>NC(=O)CC[C@H](N)C(=O)O</smiles>

Glutamine (67)<smiles>NC(Cc1ccc(O)cc1)C(=O)O</smiles>

Tyrosine (62)<smiles>N[C@@H](Cc1ccccc1)C(=O)O</smiles>

Phenylalanine (63)<smiles>N[C@@H](CC(=O)O)C(=O)O</smiles>

Aspartic acid (68)

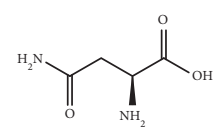

Asparagine (69)

(b)

Figure 2: Continued. 


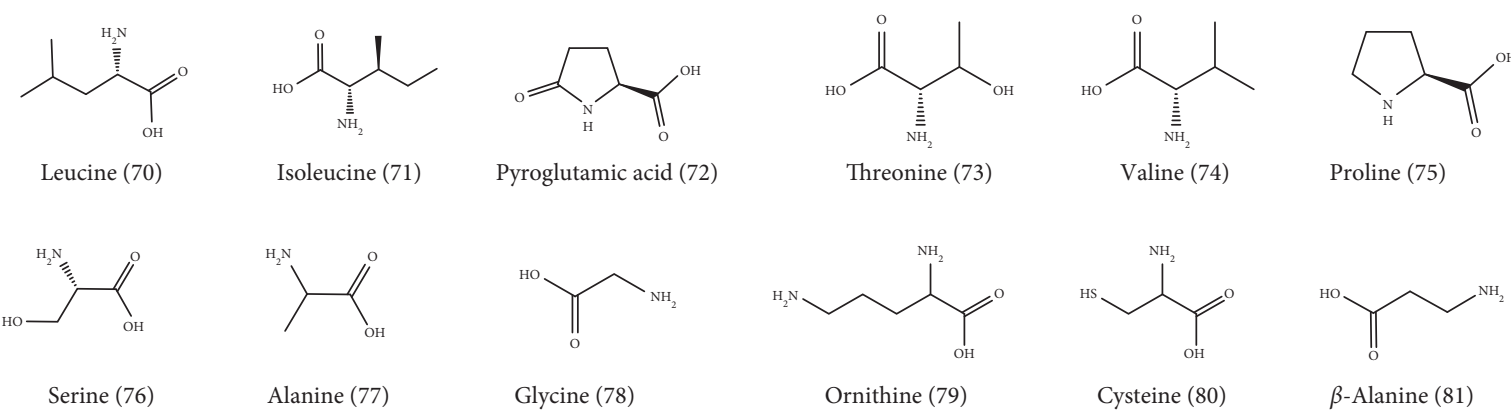<smiles>O=C(O)[C@@H](O)[C@@H](O)[C@H](O)[C@H](O)C(=O)O</smiles>

Glucaric acid (82)<smiles>O=C(O)[C@H](O)[C@@H](O)[C@H](O)[C@H](O)CO</smiles>

Gluconic acid (83)

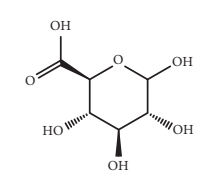

Glucuronic acid (84)<smiles>C=C(C)CC(C)(CC(=O)O)C(C)=O</smiles>

Citric acid (85)

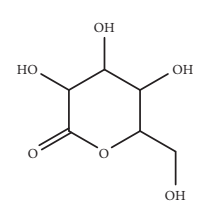

Gluconic acid-1,5-lactone (86)<smiles>O=C(O)[C@H](O)[C@@H](O)CO</smiles><smiles>O=C(O)CC(O)C(=O)O</smiles><smiles>O=C(O)CC(=O)O</smiles><smiles>O=C(O)C(=O)O</smiles><smiles>CC1=CCC2CC1C2(C)C</smiles>

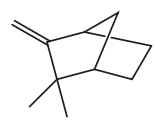

Threonic acid (87)

Malic acid (88)

Malonic acid (89)

Oxalic acid (90)

$\alpha$-Pinene (91)

2-Camphene (92)

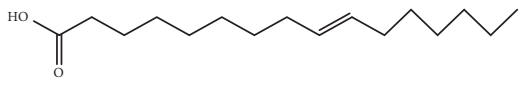

9-Hexadecenoic acid (93)

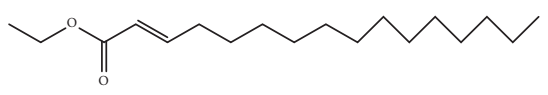

Ethyl hexadecenoic acid (95)<smiles>CCCCCC=CCC=CCCCCCCCC(=O)OCC</smiles>

Ethyl 9, 12-octadecadienoic acid (97)<smiles>CCCCCCCCCCCCCCCCCC(=O)O</smiles><smiles>CCCCCCCCCCCCC/C=C/C(=O)OC</smiles>

Methyl hexadecenoic acid (101)<smiles>CCCCCCCC/C=C/CCCCCCCC(=O)OC</smiles>

Methyl 9-octadecenoic acid (103)<smiles>CCCCCCCC/C=C/CCCCC/C=C/C(=O)O</smiles>

Octadecenoic acid (105)

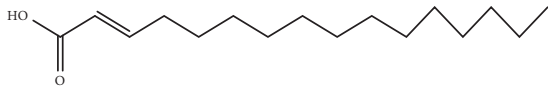

Hexadecenoic acid (94)

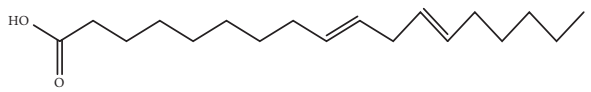

9, 12-Octadecadienoic acid (96)

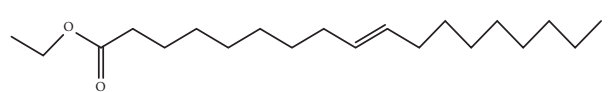

9-Ethyl octadecenate (98)
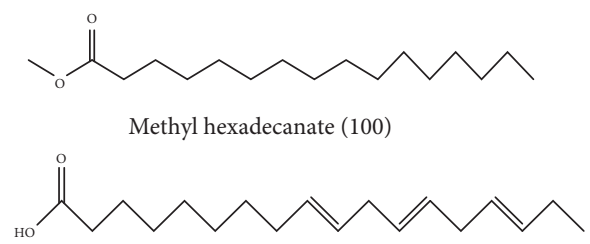

Octadeca -9, 12, 15-trienoic acid (102)

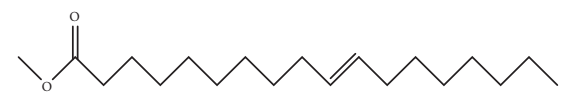

Methyl 10-octadecenoic acid (104)

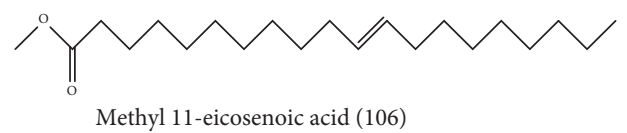

(c)

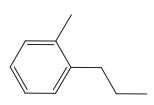

Benzene, 1-methyl-2-propyl-(108)

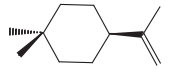

Cyclobenxene, 1-methyl-4(1-methylethenyl)-(109)

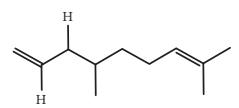

(E)-3, 7-Dimethyl-2, 6octadienal (110)

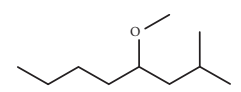

Methy 2-methyl-octanate (111)

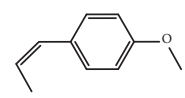

Benzene, 1-methoxy-4

(1-propenyl)-(112)

FIgURE 2: Continued. 


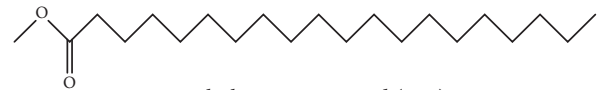

Methyl eicosenoic acid (107)

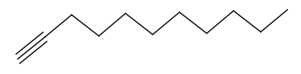

1-Undecyne (114)

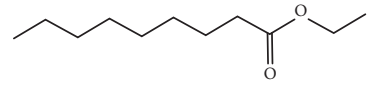

Ethyl nonanoate (115)

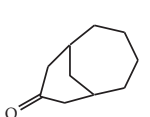

Bicyclo $(4,3,1)$ decan-10-one (117)

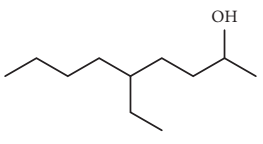

5-Ethyl-2-nonanol (118)<smiles>CC(C)CCC[C@H](C)CCCO</smiles>

4, 8-Dimethyl-1-nonanol (119)

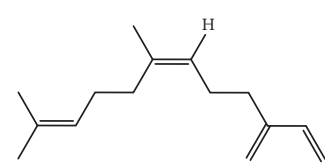

(Z)- $\beta$-Farnesene (120)<smiles>C=C/C(C)=C/C/C=C/C(C)CCC=C(C)C</smiles><smiles>CC(C)(C)C1=CC(=O)C=C(C(C)(C)C)C1=O</smiles><smiles>CC(=O)/C=C/C1=C(C)CCCC1(C)C</smiles>

$\beta$-Lonone (123)

trans- $\beta$-Farnesene (121)

2, 6-Di-T-butyl-1, 4-benzoquinone (122)<smiles>C=C1CCCC(C)(C)[C@]12CC=C(C)CC2</smiles>

$\beta$-Chamigrene (125)

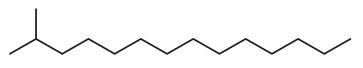

Ethyl dodecanoate (126)
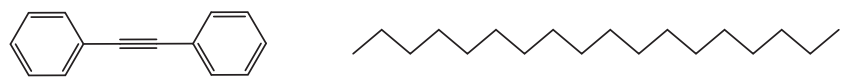

Hexadecane (127)

Methyl tetradecanoate (128) Ben

2-Penddecanone, 6, 10, 14-trimetyl-(131)

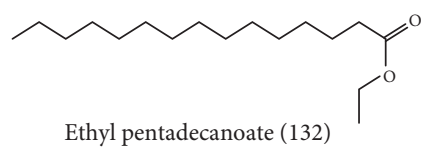

Ethyl pentadecanoate (132)

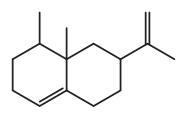

(1, 2, 3, 5, 6,7,8a-octahydro1, 8a-demethyl-7-(1-methyethyl)-, [1R$(1 \alpha, 7 \beta, 8 \mathrm{a} \alpha)]-(124)$
৩N

Eicosane (134)

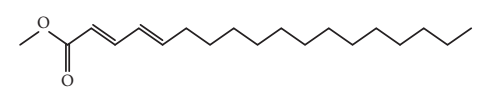

Methyl octadecadienoate (135)

Octadecane (130)

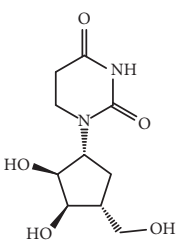

Uridine (142)

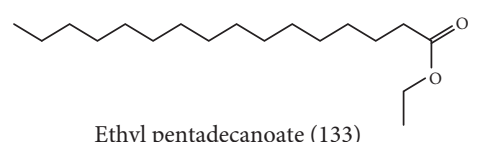

Ethyl pentadecanoate (133)

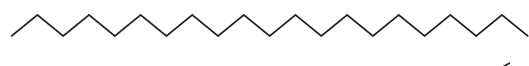

Heneicosane (136)<smiles>CCCCCCCCCCCCCCCCCC(=O)OCC</smiles>

Ethyl octadecadienoate (137)

Ethyl octadecanoate (138)

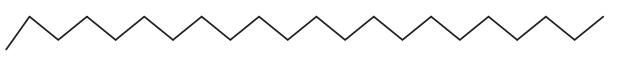

Docosane (139)

$\mathrm{NO}_{\mathrm{NH}_{2}}^{\mathrm{HO}}$

Ethanolamine (141)

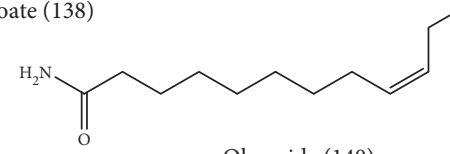

Oleamide (140)<smiles>O=C1Cc2c([nH]c(=O)[nH]c2=O)N1</smiles>

Uric acid (143)<smiles>OCCO</smiles>

Ethylene glycol (148)<smiles>Nc1nc2nc[nH]c2c(=O)[nH]1</smiles>

Guanine (144)<smiles>Nc1ncnc2nc[nH]c12</smiles>

Adenine (145)<smiles>NCCCCNCCCN</smiles>

Spermidine (146)

spe<smiles>Cc1c[nH]c(=O)[nH]c1=O</smiles>

Thymine (151)

Urea (149)<smiles>C=C1NC(=C)C(NC(N)=O)N1</smiles>

Allantoin (150)<smiles>CNC(N)=O</smiles><smiles>COc1cc(CCO)ccc1O</smiles>

2-(4-Hydroxy-3methoxyphenyl)ethanol (152)<smiles>NCCCCN</smiles>

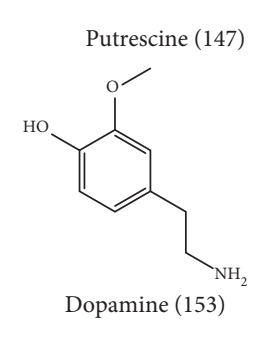

(d)

Figure 2: Chemical structures of substances within Kochiae Fructus. 
TABLe 2: Traditional uses of Kochiae Fructus (KF) referring to database "https://www.yaozh.com."

\begin{tabular}{lc}
\hline Traditional uses & Reference \\
\hline KF is used to treat frequent urination, urinary incontinence, and abnormal leucorrhea and has an effect of & Ben Cao Qiu Yuan \\
strengthening ears & Ben Cao Qiu Zhen \\
KF is used to treat frequent urination and urinary incontinence and has an effect of strengthening ears & Ben Cao Shu \\
KF has an effect of heat-clearing & Yao Jian \\
KF is used to treat skin itching and eczema and has effects of reducing swelling and improving eyesight and ears \\
KF is good for urinating and has effects of improving eyesight and ears and antiaging \\
KF is used to treat eczema & Ben Jing \\
KF has an effect for urinating & Bie Lu \\
KF is used to treat male impotence & Yao Xing Lun Yi \\
KF has an effect of reducing swelling & Ri Hua Zi Ben Cao \\
KF is used to improve urination problems, treat rubella, and abnormal vaginal discharge & Dian Nan Ben Cao \\
KF is used to treat itchy skin & Ben Cao Yuan Shi \\
KF can help urination & Ben Cao Bei Yao \\
KF is used to treat swelling and pain of the head and eyes, back pain, blood in the stool, and malignant sores & Yu Qiu Yao Jie \\
\hline
\end{tabular}

et al. showed that the underlying mechanisms of the crosstalk between apoptosis and autophagy involved ROS-related PI3K/Akt, MAPK, and NF- $\kappa \mathrm{B}$ signaling pathways, and momordin Ic simultaneously induced apoptosis and autophagy by activating these intersecting signaling pathways [27]. The summarized signal pathway is presented in Figure 3. On the contrary, momordin Ic showed a good antiinvasive activity by altering E-cadherin, VCAM-1, ICAM-1, and MMP-9, and the underlying mechanism involved PPAR $\gamma$ activation and COX-2 inhibition [30].

\subsubsection{Antiprostate Cancer Effect. The MeOH extract of KF} has shown inhibition effects on human umbilical vein endothelial cell angiogenesis and human prostate cancer cell proliferation [31]. As a member of the de-SUMOylation protease family, SUMO-specific protease 1 (SENP1) is elevated in prostate cancer (PCa) cells and is involved in PCa pathogenesis [43-46]. Momordin Ic as a novel SENP1 inhibitor could inhibit proliferation of prostate cancer cells in vitro and in vivo by inducing cell cycle arrest and apoptosis [32]. The possible mechanism is that momordin Ic could increase the sub-G1 phase cell population, increase numbers of annexin- $\mathrm{V}$ positive cells, increase active caspase-3, caspase-8, and PARP1 cleavage, and reduce cyclin B and CDK1 levels. Thus, it was considered that SENP1 may play an important role in momordin Ic-induced cell death in prostate cancer cells, even though the downstream effectors of SENP1 that mediate momordin Ic-induced apoptosis are currently unknown.

3.4. Antifungal Effect. In vitro, the water extract of $\mathrm{KF}$ showed a strong inhibition on common dermatophytes. Its minimum inhibitory concentration (MIC) on Trichophyton mentagrophytes was $3.12 \%$ and on Trichophyton rubrum, Microsporum canis, Trichophyton violaceum, and Trichophyton schoenleinii were $0.78 \%$ [33]. Wu et al. tested six KF extracts against Fusarium graminearum, Fusarium oxysporum, Monilia cinerea, Physalos porapiricola, Alternaria alternata, and Valsa mali. As a result, the water extract had the strongest inhibition effect on all six plant pathogenic bacteria with antifungal activities of more than $74.34 \%$, and the water, petroleum ether, chloroform, ethylacetate, and methanol extracts showed stronger antifungal activities against Monilia cinerea and Valsa mali than the others [34]. In addition, the saponin extract, flavone extract I (40\% alcohol eluent), flavone extract II ( $80 \%$ alcohol eluent), and lipid extract from KF were tested against Microsporum ferrugineum, Microsporum gypseum, Trichophyton schoenleini, Trichophyton mentagrophytes, Trichophyton violaceum, Trichophyton rubrum, Epidermophyton floccosum, Aspergillus fumigatus, Candida albicans, and Cryptococcus neoformans. The lipid extract showed a good antifungal effect on Microsporum ferrugineum, Microsporum gypseum, Trichophyton mentagrophytes, and Trichophyton rubrum; the saponin extract gave inhibition effects on Microsporum ferrugineum, Microsporum gypseum, Trichophyton schoenleini, Trichophyton mentagrophytes, and Trichophyton rubrum; the flavone extract I ( $40 \%$ alcohol eluent) exerted inhibition effects on Microsporum ferrugineum, Trichophyton rubrum, and Epidermophyton floccosum, whereas the flavone extract II ( $80 \%$ alcohol eluent) play an inhibitory role on Microsporum ferrugineum, Microsporum gypseum, and Trichophyton rubrum. Unfortunately, the MIC was not mentioned in this article [35]. Above all, this effect of KF supports its traditional use in gynecological infection.

3.5. Antipruritogenic Effect. The $70 \%$ ethanol extract $(200 \mathrm{mg} / \mathrm{kg})$ and methanol extract of KF $(500 \mathrm{mg} / \mathrm{kg})$ have been proved to inhibit the scratching behavior on a compound 48/80-induced pruritogenic model in male ddY mice [36]. Momordin Ic isolated from KF also exhibited an inhibition effect at a dose of $50 \mathrm{mg} / \mathrm{kg}$. Meanwhile, in an itching guinea pig model and an itching mice model, the water extract of $\mathrm{KF}$ at the concentration of $0.15 \mathrm{~g} / \mathrm{mL}$ could significantly decrease the number of itching and total time of itching within 30 minutes, indicating that KF could be used as an antipruritogenic agent [33]. These results agree with the traditional use of KF for itch. 
TABle 3: Pharmacological effects of Kochiae Fructus (KF).

\begin{tabular}{|c|c|c|c|c|}
\hline $\begin{array}{l}\text { Pharmacological } \\
\text { effect }\end{array}$ & Model & Administration & Minimal active concentration & Reference \\
\hline \multirow{11}{*}{$\begin{array}{l}\text { Anti- } \\
\text { inflammatory } \\
\text { effect }\end{array}$} & $\begin{array}{l}\text { The ddY mice in an acetic acid- } \\
\text { induced vascular permeability }\end{array}$ & $\begin{array}{l}\text { Tested drug: } 70 \% \text { ethanol extract } \\
\text { of } \mathrm{KF} \text { at the doses of } 50,200 \text {, and } \\
500 \mathrm{mg} / \mathrm{kg} \text {, p.o. } \\
\text { Positive control: indomethacin at } \\
10 \mathrm{mg} / \mathrm{kg}\end{array}$ & $200 \mathrm{mg} / \mathrm{kg}$ & {$[19]$} \\
\hline & $\begin{array}{c}\text { The ddY mice in a carrageenin- } \\
\text { induced edema }\end{array}$ & $\begin{array}{l}\text { Tested drug: } 70 \% \text { ethanol extract } \\
\text { of } \mathrm{KF} \text { at the doses of } 50,200 \text {, and } \\
500 \mathrm{mg} / \mathrm{kg} \text {, p.o. } \\
\text { Positive control: indomethacin at } \\
10 \mathrm{mg} / \mathrm{kg}\end{array}$ & $200 \mathrm{mg} / \mathrm{kg}$ & {$[19]$} \\
\hline & $\begin{array}{l}\text { The ddY mice in a compound } 48 / 80 \text { - } \\
\text { induced edema }\end{array}$ & $\begin{array}{l}\text { Tested drug: } 70 \% \text { ethanol extract } \\
\text { of KF at the doses of } 50,200 \text {, and } \\
500 \mathrm{mg} / \mathrm{kg} \text {, p.o. } \\
\text { Positive control: } \\
\text { diphenhydramine at } 50 \mathrm{mg} / \mathrm{kg}\end{array}$ & $500 \mathrm{mg} / \mathrm{kg}$ & {$[19]$} \\
\hline & $\begin{array}{l}\text { The ddY mice in a chemical } \\
\text { mediator-induced edema }\end{array}$ & $\begin{array}{l}\text { Tested drug: } 70 \% \text { ethanol extract } \\
\text { of } \mathrm{KF} \text { at the doses of } 50,200 \text {, and } \\
500 \mathrm{mg} / \mathrm{kg} \text {, p.o. } \\
\text { Positive control: cyproheptadine } \\
\text { at } 2 \mathrm{mg} / \mathrm{kg} \text {; diphenhydramine at } \\
50 \mathrm{mg} / \mathrm{kg}\end{array}$ & $200 \mathrm{mg} / \mathrm{kg}$ & [19] \\
\hline & $\begin{array}{l}\text { The isolated ileum of guinea pig in a } \\
\text { histamine-induced contraction }\end{array}$ & $\begin{array}{c}\text { Tested drug: } 70 \% \text { ethanol extract } \\
\text { of KF at the doses of } 10,50,100 \text {, } \\
\text { and } 300 \mu \mathrm{g} / \mathrm{mL}\end{array}$ & $220 \mu \mathrm{g} / \mathrm{mL}$ & {$[19]$} \\
\hline & $\begin{array}{l}\text { The ddY mice in an arachidonic acid- } \\
\text { induced edema }\end{array}$ & $\begin{array}{l}\text { Tested drug: } 70 \% \text { ethanol extract } \\
\text { of KF at the doses of } 50,200 \text {, and } \\
500 \mathrm{mg} / \mathrm{kg} \text {, p.o. } \\
\text { Positive control: phenidone at } \\
20 \mathrm{mg} / \mathrm{kg} \text {, i.v. }\end{array}$ & $500 \mathrm{mg} / \mathrm{kg}$ & [19] \\
\hline & $\begin{array}{l}\text { A picryl chloride-induced ear } \\
\text { inflammatory model in ICR mice }\end{array}$ & $\begin{array}{l}\text { Tested drug: } 70 \% \text { ethanol extract } \\
\text { of } \mathrm{KF} \text { at the doses of } 100,200 \text {, and } \\
500 \mathrm{mg} / \mathrm{kg} \text {, i.g. } \\
\text { Positive control: prednisone at } \\
50 \mathrm{mg} / \mathrm{kg}\end{array}$ & $500 \mathrm{mg} / \mathrm{kg}$ & {$[20]$} \\
\hline & $\begin{array}{l}\text { A dinitrochlorobenzene-induced } \\
\text { allergic contact dermatitis model in } \\
\text { rats }\end{array}$ & $\begin{array}{l}\text { Tested drug: total flavonoids of KF } \\
\text { at the doses of } 100 \text { and } 200 \mathrm{mg} / \mathrm{kg} \text {. } \\
\text { p.o. } \\
\text { Positive control: sodium } \\
\text { prednisolone acetate at } 2.5 \mu \mathrm{g} / \mathrm{mL}\end{array}$ & $100 \mathrm{mg} / \mathrm{kg}$ & {$[21]$} \\
\hline & $\begin{array}{l}\text { A DNFB-induced contact dermatitis } \\
\text { model in mice }\end{array}$ & $\begin{array}{l}\text { Tested drug: methanol extract of } \\
\text { KF at the doses of } 30,100 \text {, and } \\
300 \mu \mathrm{g} / \text { ear for external use } \\
\text { Positive control: dexamethasone } \\
\text { at } 75 \mu \mathrm{g} / \text { ear }\end{array}$ & $100 \mu \mathrm{g} / \mathrm{ear}$ & {$[22]$} \\
\hline & $\begin{array}{l}\text { The ddY mice in a carrageenin- } \\
\text { induced edema }\end{array}$ & $\begin{array}{c}\text { Tested drug: momordin Ic at the } \\
\text { doses of } 20,50 \text {, and } 100 \mathrm{mg} / \mathrm{kg} \text {, } \\
\text { p.o. } \\
\text { Positive control: indomethacin at } \\
10 \mathrm{mg} / \mathrm{kg}\end{array}$ & $20 \mathrm{mg} / \mathrm{kg}$ & [19] \\
\hline & LPS-stimulated RAW 264.7 cell line & $\begin{array}{c}\text { Tested drug: } 20 \text {-hydroxyecdysone, } \\
\text { momordin Ic, and oleanolic acid } \\
\text { with various concentrations ( } 6.25 \text {, } \\
12.5 \text {, or } 25 \mu \mathrm{M}) \\
\text { Positive control: indomethacin at } \\
2.5 \mathrm{ng} / \mathrm{mL}\end{array}$ & $6.25 \mu \mathrm{M}$ & {$[5]$} \\
\hline
\end{tabular}


TABle 3: Continued.

\begin{tabular}{|c|c|c|c|c|}
\hline $\begin{array}{l}\text { Pharmacological } \\
\text { effect }\end{array}$ & Model & Administration & Minimal active concentration & Reference \\
\hline \multicolumn{5}{|c|}{ Hypoglycemic effect } \\
\hline & $\begin{array}{l}\text { Normal, alloxan-induced } \\
\text { hyperglycemic and insulin-induced } \\
\text { hypoglycemic mice }\end{array}$ & $\begin{array}{l}\text { Tested drug: } n \text {-butanol extract of } \\
\text { KF at the doses of } 25 \text { and } 50 \mathrm{mg} / \\
\text { kg, p.o. }\end{array}$ & $25 \mathrm{mg} / \mathrm{kg}$ & {$[23]$} \\
\hline & $\begin{array}{c}\text { Examining the activity of } \\
\alpha \text {-glucosidase in rat intestine in vitro }\end{array}$ & $\begin{array}{l}\text { Tested drug: } n \text {-butanol extract of } \\
\mathrm{KF} \text { at the doses of } 62.5,125,250 \text {, } \\
\text { and } 500 \mu \mathrm{g} / \mathrm{mL} \\
\text { Positive control: acarbose at } 2 \mu \mathrm{g} / \\
\mathrm{mL}\end{array}$ & $125 \mu \mathrm{g} / \mathrm{mL}$ & {$[23]$} \\
\hline & $\begin{array}{l}\text { Examining the ability of glucose } \\
\text { absorption in rat intestine in vitro }\end{array}$ & $\begin{array}{c}\text { Tested drug: } n \text {-butanol extract of } \\
\mathrm{KF} \text { at the doses of } 0,100,200,400, \\
\text { and } 800 \mu \mathrm{g} / \mathrm{mL}\end{array}$ & $100 \mu \mathrm{g} / \mathrm{mL}$ & {$[23]$} \\
\hline & $\begin{array}{l}\text { Testing the propulsive function of } \\
\text { small intestine in normal, } \\
\text { fenfluramine-treated, dopamine- } \\
\text { treated, acetic acid-treated, and N } \omega \text { - } \\
\text { nitro-L-arginine-treated rats }\end{array}$ & $\begin{array}{l}\text { Tested drug: } n \text {-butanol extract of } \\
\text { KF at the doses of } 25 \text { and } 50 \mathrm{mg} / \\
\text { kg, p.o. }\end{array}$ & $50 \mathrm{mg} / \mathrm{kg}$ & {$[24]$} \\
\hline & Testing the gastric emptying in rats & $\begin{array}{l}\text { Tested drug: oleanolic acid 3-O- } \\
\text { glucuronide and momordin Ic at } \\
\text { the doses of } 12.5,25 \text {, and } 50 \mathrm{mg} / \\
\mathrm{kg} \text {, p.o. } \\
\text { Positive control: atropine sulfate } \\
\text { at } 10 \mathrm{mg} / \mathrm{kg}\end{array}$ & $\begin{array}{l}25 \text { and } 12.5 \mathrm{mg} / \mathrm{kg} \text {, } \\
\text { respectively }\end{array}$ & {$[25]$} \\
\hline & $\begin{array}{l}\text { Testing the glucose uptake in rat } \\
\text { small intestine in vitro }\end{array}$ & $\begin{array}{l}\text { Tested drug: oleanolic acid 3-O- } \\
\text { glucuronide and momordin Ic at } \\
\text { the doses of } 0,5,50 \text {, and } 500 \mu \mathrm{M} \\
\text { Positive control: phlorizin at } 1 \mu \mathrm{M}\end{array}$ & 50 and $5 \mu \mathrm{M}$, respectively & {$[25]$} \\
\hline & $\begin{array}{l}\text { Gastric emptying test on } 1.5 \% \\
\text { carboxymethyl cellulose sodium salt } \\
\text { test meal-loaded mice, } 40 \% \text { glucose } \\
\text { test meal-loaded mice, milk test meal- } \\
\text { loaded mice, and } 60 \% \text { ethanol test } \\
\text { meal-loaded mice }\end{array}$ & $\begin{array}{l}\text { Tested drug: momordin Ic in the } \\
\text { dose range of } 12.5-50 \mathrm{mg} / \mathrm{kg} \text {, p.o. }\end{array}$ & $50 \mathrm{mg} / \mathrm{kg}$ & {$[3]$} \\
\hline \multicolumn{5}{|l|}{ Anticancer effects } \\
\hline \multirow{5}{*}{ Antiliver cancer } & $\begin{array}{l}\text { Inducing apoptosis of human } \\
\text { hepatocyte carcinoma HepG2 cells }\end{array}$ & $\begin{array}{l}\text { Tested drug: momordin Ic in the } \\
\text { concentration range of } 10-30 \mu \mathrm{M}\end{array}$ & $15 \mu \mathrm{M}$ & {$[26]$} \\
\hline & $\begin{array}{l}\text { Inducing autophagy of human } \\
\text { hepatocyte carcinoma HepG2 cells }\end{array}$ & $\begin{array}{l}\text { Tested drug: momordin Ic in the } \\
\text { dose range of } 5-20 \mu \mathrm{M}\end{array}$ & $10 \mu \mathrm{M}$ & {$[27]$} \\
\hline & $\begin{array}{l}\text { Inducing apoptosis of human } \\
\text { hepatocyte carcinoma HepG2 cells }\end{array}$ & $\begin{array}{l}\text { Tested drug: momordin Ic in the } \\
\text { dose range of } 5,10 \text {, and } 15 \mu \mathrm{M}\end{array}$ & $5 \mu \mathrm{M}$ & [28] \\
\hline & $\begin{array}{l}\text { Suppressing invasion of human } \\
\text { hepatocyte carcinoma HepG2 cells }\end{array}$ & $\begin{array}{c}\text { Tested drug: momordin Ic at the } \\
\text { dose of } 10 \mu \mathrm{M}\end{array}$ & $10 \mu \mathrm{M}$ & [29] \\
\hline & $\begin{array}{l}\text { Inhibiting migration and invasion of } \\
\text { human hepatocyte carcinoma HepG2 } \\
\text { cells }\end{array}$ & $\begin{array}{l}\text { Tested drug: momordin Ic in the } \\
\text { dose range of } 1-10 \mu \mathrm{M}\end{array}$ & $5 \mu \mathrm{M}$ & {$[30]$} \\
\hline \multirow{2}{*}{$\begin{array}{l}\text { Antiprostate } \\
\text { cancer }\end{array}$} & $\begin{array}{l}\text { VEGF-induced angiogenesis in } \\
\text { human umbilical vein endothelial } \\
\text { cells and proliferation in prostate } \\
\text { cancer cells }\end{array}$ & $\begin{array}{c}\text { Tested drug: methanol extract of } \\
K F \text { in the dose range of } 10-20 \mu \mathrm{g} / \\
\mathrm{mL} \text { and } 10-250 \mu \mathrm{g} / \mathrm{mL}, \\
\text { respectively }\end{array}$ & $\begin{array}{l}20 \mu \mathrm{g} / \mathrm{mL} \text { and } 100 \mu \mathrm{g} / \mathrm{mL} \text {, } \\
\text { respectively }\end{array}$ & {$[31]$} \\
\hline & $\begin{array}{l}\text { Testing the SUMO-specific protease } 1 \\
\text { in prostate cancer cells and a } \\
\text { xenograft PC } 3 \text { tumor mouse model }\end{array}$ & $\begin{array}{c}\text { Tested drug: momordin Ic at the } \\
\text { dose of } 6.25,12.5 \text {, and } 25 \mu \mathrm{M} \text { and } \\
10 \mathrm{mg} / \mathrm{kg} / \text { day, i.p. For } 20 \text { days, } \\
\text { respectively }\end{array}$ & $\begin{array}{c}\mathrm{IC}_{50} \text { was } 15.37 \mu \mathrm{M} \text { and } 10 \mathrm{mg} / \\
\mathrm{kg} / \text { day }\end{array}$ & {$[32]$} \\
\hline
\end{tabular}


TABle 3: Continued.

\begin{tabular}{|c|c|c|c|c|}
\hline $\begin{array}{l}\text { Pharmacological } \\
\text { effect }\end{array}$ & Model & Administration & Minimal active concentration & Reference \\
\hline Antifungal effect & $\begin{array}{l}\text { In vitro for Trichophyton } \\
\text { mentagrophytes, Trichophyton } \\
\text { rubrum, Microsporum canis, } \\
\text { Trichophyton violaceum, and } \\
\text { Trichophyton schoenleinii } \\
\text { Inhibiting the growing of Fusarium } \\
\text { graminearum, Fusarium oxysporum, } \\
\text { Monilia cinerea, Physalos } \\
\text { porapiricola, Alternaria alternata, } \\
\text { and Valsa mali } \\
\text { In vitro for Microsporum } \\
\text { ferrugineum, Microsporum gypseum, } \\
\text { Trichophyton schoenleini, } \\
\text { Trichophyton mentagrophytes, } \\
\text { Trichophyton violaceum, } \\
\text { Trichophyton rubrum, } \\
\text { Epidermophyton floccosum, } \\
\text { Aspergillus fumigatus, Candida } \\
\text { albicans, Cryptococcus neoformans }\end{array}$ & $\begin{array}{l}\text { Tested drug: water extract of } \mathrm{KF} \text { in } \\
\text { the concentration range of } 0.04 \%- \\
25 \% \\
\text { Tested drug: water, petroleum } \\
\text { ether, chloroform, ethylacetate, } \\
\text { and methanol extract of } K F \text { with } \\
\text { the concentration of dose } 1 \mathrm{mg} / \\
15 \mathrm{~mL}\end{array}$ & $\begin{array}{l}\text { All samples have the } \\
\text { antifungal effect on } \\
\text { Microsporum ferrugineum and } \\
\text { Trichophyton rubrum, but the } \\
\text { concentration was not } \\
\text { mentioned }\end{array}$ & {$[34]$} \\
\hline Antipruritogenic & $\begin{array}{l}\text { A compound } 48 / 80 \text {-induced } \\
\text { pruritogenic model in male ddY mice }\end{array}$ & $\begin{array}{c}\text { Tested drug: } 70 \% \text { ethanol extract } \\
\text { of } \mathrm{KF} \text { at the dose of } 200 \text { and } \\
500 \mathrm{mg} / \mathrm{kg} \text {, p.o. } \\
\text { Tested drug: methanol extract of } \\
\text { KF at the dose of } 50,200 \text {, and } \\
500 \mathrm{mg} / \mathrm{kg} \text {, p.o. } \\
\text { Tested drug: momordin Ic at the } \\
\text { dose of } 20,50 \text {, and } 100 \mathrm{mg} / \mathrm{kg} \text {, p.o. } \\
\text { Positive control: } \\
\text { diphenhydramine at } 20 \mathrm{mg} / \mathrm{kg} \\
\text { Tested drug: water extract of KF at } \\
\text { the concentration of } 0.15 \mathrm{~g} / \mathrm{mL} \text {, } \\
0.3 \mathrm{~g} / \mathrm{mL} \text {, and } 0.6 \mathrm{~g} / \mathrm{mL} \text { for } \\
\text { external use } \\
\text { Positive control: cyproheptadine }\end{array}$ & $500 \mathrm{mg} / \mathrm{kg}$ & {$[36]$} \\
\hline Antinociceptive $e$ & $\begin{array}{l}\text { The ddY mice in an acetic acid- } \\
\text { induced writhing test }\end{array}$ & $\begin{array}{c}\text { Tested drug: } 70 \% \text { ethanol extract } \\
\text { of } \mathrm{KF} \text { at the dose of } 50,200 \text {, and } \\
500 \mathrm{mg} / \mathrm{kg} \text {, p.o. } \\
\text { Tested drug: momordin Ic at the } \\
\text { dose of } 20,50 \text {, and } 100 \mathrm{mg} / \mathrm{kg} \text {, p.o. } \\
\text { Positive control: aspirin at } \\
200 \mathrm{mg} / \mathrm{kg}\end{array}$ & $500 \mathrm{mg} / \mathrm{kg}$ & {$[19]$} \\
\hline Inhibition effect & $\begin{array}{l}\text { DTH models upon challenge with } \\
\text { SRBC or PC }\end{array}$ & $\begin{array}{l}\text { Tested drug: } 70 \% \text { ethanol extract } \\
\text { of KF at the dose of } 100,200 \text {, and } \\
500 \mathrm{mg} / \mathrm{kg} \text {. } \\
\text { Positive control: prednisone at } \\
50 \mathrm{mg} / \mathrm{kg} \\
\text { Tested drug: total saponins extract } \\
\text { of } \mathrm{KF} \text { at the dose of } 50,100 \text {, and } \\
200 \mu \mathrm{g} / \mathrm{mL} \\
\text { Positive control: cyproheptadine } \\
\text { at } 20 \mathrm{mg} / \mathrm{kg}\end{array}$ & $200 \mathrm{mg} / \mathrm{kg}$ & {$[20]$} \\
\hline Myocardial prote & $\begin{array}{l}\text { A furazolidone-induced dilated } \\
\text { cardiomyopathy model in Wistar rats }\end{array}$ & $\begin{array}{l}\text { Tested drug: water extract of KF at } \\
\text { the dose of } 5 \text { and } 20 \mathrm{mg} / \mathrm{g} \text {, p.o. } \\
\text { Negative model: water }\end{array}$ & $20 \mathrm{mg} / \mathrm{g}$ & {$[37]$} \\
\hline
\end{tabular}


TABle 3: Continued.

\begin{tabular}{|c|c|c|c|c|}
\hline $\begin{array}{l}\text { Pharmacological } \\
\text { effect }\end{array}$ & Model & Administration & Minimal active concentration & Reference \\
\hline \multicolumn{5}{|c|}{ Hepatoprotective effect } \\
\hline & $\begin{array}{l}\text { Carbon tetrachloride-induced liver } \\
\text { damage in rats }\end{array}$ & $\begin{array}{l}\text { Tested drug: momordin Ic and } \\
\text { oleanolic acid at the dose of } \\
30 \mathrm{mg} / \mathrm{kg} / \text { day for } 14 \text { days, p.o. } \\
\text { Negative control: saline }\end{array}$ & $30 \mathrm{mg} / \mathrm{kg} /$ day for 14 days & {$[38]$} \\
\hline \multicolumn{5}{|c|}{ Protecting gastric mucosal lesions } \\
\hline & $\begin{array}{l}\text { Ethanol-induced gastric mucosal } \\
\text { lesions in rats and indomethacin- } \\
\text { induced gastric mucosal lesions in } \\
\text { rats }\end{array}$ & $\begin{array}{l}\text { Tested drug: momordin Ic in the } \\
\text { dose range of } 2.5-50 \mathrm{mg} / \mathrm{kg} \text {, p.o. } \\
\text { Positive control: omeprazole at } 20 \\
\text { or } 5 \mathrm{mg} / \mathrm{kg} \text {, respectively }\end{array}$ & $5 \mathrm{mg} / \mathrm{kg}$ & [39] \\
\hline \multicolumn{5}{|c|}{ Suppressing osteoclastogenesis } \\
\hline & $\begin{array}{l}\text { A cocultured system and a RANKL- } \\
\text { induced osteoclast precursor system }\end{array}$ & $\begin{array}{l}\text { Tested drug: momordin Ic in the } \\
\text { dose range of } 0.1-5 \mu \mathrm{M}\end{array}$ & $0.5 \mu \mathrm{M}$ & {$[40]$} \\
\hline \multicolumn{5}{|l|}{ Antibacteria effect } \\
\hline & $\begin{array}{l}\text { Minimum inhibitory concentration } \\
\text { (MIC) test on Escherichia coli }\end{array}$ & $\begin{array}{l}\text { Tested drug: oleanolic acid in the } \\
\text { dose range of } 15.6-4000 \mu \mathrm{g} / \mathrm{mL}\end{array}$ & $31.3 \mu \mathrm{g} / \mathrm{mL}$ & {$[41]$} \\
\hline
\end{tabular}

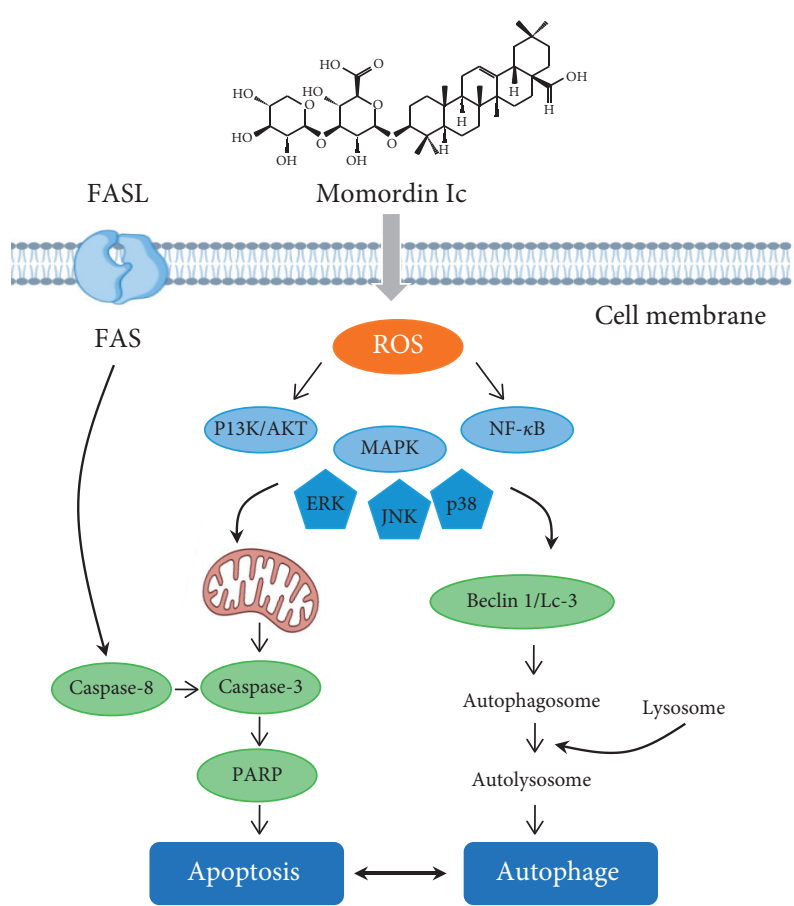

Figure 3: The signal pathways of antiliver cancer effect of momordin Ic.

3.6. Others. The inhibition effect of hypersensitivity of $70 \%$ ethanol and total saponin extracts from KF has been tested on DTH models upon challenge with SRBC or PC. As a result, the $70 \%$ ethanol extract produced a concentrationdependent reduction on immediate and delayed-type hypersensitivity, while total saponins extract showed an inhibitory tendency at $200 \mathrm{mg} / \mathrm{kg}$ [20]. This effect might have a close relationship with its stabilization of mast cell membrane, reduction of release of anaphylactic mediators, and anti-inflammatory activities [20]. The water extract of KF at the dose of $20 \mathrm{mg} / \mathrm{g}$ could reverse the imbalance of Th1/Th2 cell in rat model with dilated cardiomyopathy, which reduced the damage of immune response to myocardium and protected the heart function. Momordin Ic gave a protective effect on gastric mucosal lesions [37]. Kim et al. discovered that momordin Ic has a hepatoprotective effect against $\mathrm{CCl}_{4}$-induced liver damage because it could enhance the hepatic antioxidant defense system [38]. Meanwhile, as an AP-1 inhibitor, momordin Ic could downregulate NF- $\kappa \mathrm{B}$ activation as well as AP-1 activation, which plays a key role in osteoclast differentiation, by inhibiting $\mathrm{I} \kappa \mathrm{B}$ degradation and c-Fos expression, respectively. Therefore, momordin Ic has high potential to be a good candidate for controlling bone disorders in the future [40]. In addition, the extracts of $\mathrm{KF}$, including total flavonoid, saponin, and phenolic, were proved to have a good antioxidant activity by measuring free radical scavenging activities with ABTS, DPPH, or FARP assay [47-50]. Scavenging free radicals play a key role in aging and inflammation [51]. Therefore, its antioxidant effects need to be further studied on these aspects.

The studies on toxicity of KF are scarce. Although KF is almost nontoxic in traditional use, the animal death was found when a large dosage is used. According to a toxicological study, when KM mice were orally administrated with water extract of $\mathrm{KF}$ in a dose range from $4.5 \mathrm{mg} / \mathrm{kg}$ to $9.4 \mathrm{mg} / \mathrm{kg}$, the median lethal dose $\left(\mathrm{LD}_{50}\right)$ was $7.15 \pm 0.03 \mathrm{~g} / \mathrm{kg}$ [35]. Therefore, the toxic and side effects of KF should be paid more attention in its clinical application.

\section{Quality Control}

The quality of herbal medicines is the key for its clinical efficacy and safety, and hence, establishing a quality control system is the premise of its clinical application. The quality control of KF was focused on quantitative analysis of components by a series of analytical methods, such as ultraviolet-visible detector (UV), gas chromatography-mass spectrometry (GC-MS), and high-performance liquid chromatography-evaporative light scattering detector (HPLC-ELSD). Since the main component of KF is triterpenoid saponin, whose UV absorption is poor, 
researchers incline to use ELSD. Xia et al. developed a method to determine the content of momordin Ic in KF and the content of momordin Ic was $0.83 \%-0.21 \%$ in four kinds of marketed KF [52]. Moreover, they used HPLC-ELSD and colorimetric methods to determine the content of momordin Ic and total saponins, respectively, in $\mathrm{KF}$ at different collecting times or from eleven places in China $[53,54]$. Except for saponin, there also established an HPLC method with good stability and reproducibility to simultaneously determine the content of rutin and quercetin in KF [55]. Currently, the fingerprint derived from HPLC has been an acknowledged method to control the quality of traditional Chinese medicine and botanical medicine. An HPLC fingerprint method was applied to 10 batches of KF purchased from Shandong, China. According to the cluster analysis, 19 common peaks of fingerprint were found, and 10 batches could be divided into 3 groups related to their origins [56]. The results showed that this method could differentiate samples from different geographical origins or processing methods. Nevertheless, there were few compounds quantified as mark compounds for the quality control of KF, and it is in urgent need of a comprehensive quantification method to further ensure the quality control.

\section{Pharmacokinetics}

Momordin Ic is a representative pharmacologically active ingredient and quality control marker of KF, and its pharmacokinetic property has attracted attentions. Yan et al. developed and validated a highly selective and sensitive method based on ultraperformance liquid chromatography-tandem mass spectrometry (UPLC-MS/MS) for routine analysis of momordin Ic in rat plasma. After intravenous administration of momordin Ic at $0.52,1.56$, and $4.67 \mathrm{mg} / \mathrm{kg}$ in rats, the $\mathrm{AUC}_{\text {last }}$ (area under the concentration-time curve from time 0 to $t$ hours postodose) values were $1864.17 \pm 431.01,5466.00 \pm 889.86$, and $16890.45 \pm$ $3028.64 \mathrm{ng} \mathrm{h} / \mathrm{mL}$, respectively, which was consistent with linear pharmacokinetic characteristics. The elimination half-life $\left(t_{1 / 2}\right)$ values were $1.22 \pm 0.39,1.14 \pm 0.10$, and $1.83 \pm 0.39 \mathrm{~h}$, respectively [4]. However, there are few studies on the pharmacokinetic of other substances within KF and on the interaction between substances during their ADME in vivo.

\section{Conclusion and Perspectives}

Recent pharmacology studies showed anti-inflammatory, antifungal, antiallergic, and antipruritogenic effects of KF, which supports the traditional clinical applications including the treatment of diseases in the skin, eye, and urinary tract in China, Korea, and Japan. Interestingly, the anticancer, hypoglycemic, and hepatoprotective effects of KF were also tested. Besides, the potential mechanisms of some effects were also elucidated. However, there are few toxicology studies on KF, which may be necessary for its better application as a medicine or a food. A total of 25 triterpenoids, 13 flavonoids, 22 carbohydrates, 21 amino acids, 9 organic acid, 49 essential oils, and 14 heterocyclics within KF have been reported. Momordin Ic is a main substance, and it is usually used as a phytochemical marker for the quality control of KF. The pharmacological effects were achieved by the chemical constituents within KF. Hence, the interrelationship between compounds and pharmacological activities should be further studied. The pharmacokinetics of KF was lack, and a range of pharmacokinetic studies on its active compounds are needed to provide comprehensive data for clinical application. Altogether, this review extensively summarized phytochemistry, pharmacology, toxicity, quality control, and pharmacokinetic studies on KF to provide information for its further research and clinical applications.

\section{Data Availability}

The data used to support the findings of this study are available from the corresponding author upon request.

\section{Disclosure}

The funding sponsors had no intervention in the design of the study, collection, analyses, and interpretation of data, writing the manuscript, and also the decision to publish the experiment results.

\section{Conflicts of Interest}

The authors declare that there are no conflicts of interest.

\section{Authors' Contributions}

Wei Zou and Zhong Tang contributed equally.

\section{Acknowledgments}

This research was funded by the Hunan Provincial Science and Technology Department (Grant nos. 2018SK50501, 2019JJ30013, and 2020RC3065) and Hunan Administration of Traditional Chinese Medicine (Grant no. 202020).

\section{References}

[1] Flora of China Editorial Committee and the Chinese Academy of Sciences, Flora of China, Beijing Science Press, Beijing, China, 1986.

[2] L. I. Pei-Yuan, H. Li-Ni, S. U. Wei, D. Ling-Yu, P. Mei-Ping, and Z. Zhi-Xiang, "Radical scavenging activity and total phenolics content of Kochia scoparia," Hub Agricultural Ences, vol. 55, no. 11, pp. 2899-2901, 2016.

[3] H. Matsuda, Y. Li, J. Yamahara, and M. Yoshikawa, "Inhibition of gastric emptying by triterpene saponin, momordin Ic, in mice: roles of blood glucose, capsaicin-sensitive sensory nerves, and central nervous system," Journal of Pharmacology and Experimental Therapeutics, vol. 289, no. 2, pp. 729-734, 1999.

[4] H. Yan, Y. Song, W. Zhou, and S. Zhang, "A selective and sensitive method based on UPLC-MS/MS for quantification of momordin Ic in rat plasma: application to a pharmacokinetic study," Journal of Pharmaceutical and Biomedical Analysis, vol. 115, pp. 196-200, 2015.

[5] S. R. Yoo, S. J. Jeong, N. R. Lee, H. K. Shin, and C. S. Seo, "Quantification analysis and in vitro anti-inflammatory effects of 20-hydroxyecdysone, momordin Ic, and oleanolic acid 
from the fructus of Kochia scoparia," Pharmacognosy Magazine, vol. 13, no. 51, pp. 339-344, 2017.

[6] L. U. Xiang-Hong, X. U. Xiang-Dong, F. U. Hong-Wei, B. Chen, J. K. Tian, and L. Zhang, "Study on chemical constituents of Kochia scoparia," Journal of Chinese Medicinal Materials, vol. 36, no. 6, pp. 921-924, 2012.

[7] H. Matsuda, Y. Dai, Y. Ido et al., "Studies on Kochiae Fructus. V. Antipruritic effects of oleanolic acid glycosides and the structure-requirement," Biological \& Pharmaceutical Bulletin, vol. 21, no. 11, pp. 1231-1233, 1998.

[8] M. Yoshikawa, Y. Dai, H. Shimada et al., "Studies on Kochiae Fructus. II. On the Saponin constituents from the fruit of Chinese Kochia Scoparia (Chenopodiaceae): chemical structures of Kochianosides I, II, III, and IV," Chemical \& Pharmaceutical Bulletin, vol. 45, 2010.

[9] W. Hao, F. Chun-Lin, W. Bei, D. Yue, and Y. E. Wen-Cai, Triterpene and Saponins from Kochia Scoparia, Chinese Journal of Natural Medicines, Beijing, China, 2003.

[10] X. U. Yun-Hui, H. Huang, Z. X. Guo, N. Zhang, D. Y. Kong, and M. L. Hua, Chemical Constituents of Antifungal Extract from Kochiae Fructus, Chinese Traditional Patent Medicine, Beijing, China, 2012.

[11] Y. Wen, Y. Chen, Z. Cui, J. Li, and Z. Wang, "Triterpenoid glycosides from the fruits ofKochia scoparia," Planta Medica, vol. 61, no. 5, pp. 450-452, 1995.

[12] Y.-H. Xu, H. Huang, N. Zhang, D.-Y. Kong, and M.-L. Hua, "Studies on the flavone glycosides from fructus kochiae," Journal of Asian Natural Products Research, vol. 16, no. 2, pp. 141-147, 2014.

[13] A. J. Houlihan, P. Conlin, and J. C. Chee-Sanford, "Watersoluble exudates from seeds of Kochia scoparia exhibit antifungal activity against Colletotrichum graminicola," PLoS One, vol. 14, no. 6, 2019.

[14] M. Yang, J. Li, J. Cai, and S. Yang, "Supercritical CO2 extraction and GC-MS analysis of Kochia Fructus oil," Zhong Yao Cai, vol. 26, no. 7, p. 494, 2003.

[15] Y. Wen, Z. Wang, and C. Xu, "Study on the constituents of essential oil from Kochiae Fructus," Zhong Yao Cai, vol. 1, no. 2, pp. 29-31, 1992.

[16] J. B. Chang, M. E. Lane, M. Yang, and M. Heinrich, "A hexaherbal TCM decoction used to treat skin inflammation: an LC-MS-based phytochemical analysis," Planta Medica, vol. 82, no. 11-12, pp. 1134-1141, 2016.

[17] N. Ma and X. Ma, "Dietary amino acids and the gutmicrobiome-immune Axis: physiological metabolism and therapeutic prospects," Comprehensive Reviews in Food Science and Food Safety, vol. 18, no. 1, pp. 221-242, 2019.

[18] Y. Zhang, R. Wang, C. Wang, and S. Bao, "Research pregress on traditional Chinese medicine Kochia scoparia (L.) Schrad," Chinese Medicine Journal of Research and Practice, vol. 30, no. 1, pp. 84-86, 2016.

[19] H. Matsuda, Y. Dai, Y. Ido, M. Yoshikawa, and M. Kubo, "Studies on kochiae fructus. IV. Anti-allergic effects of 70\% ethanol extract and its component, momordin Ic from dried fruits of Kochia scoparia L," Biological \& Pharmaceutical Bulletin, vol. 20, no. 11, pp. 1165-1170, 1997.

[20] Y. Dai, Y. F. Xia YufengXia, and H. B. Chen HaibiaoChen, "Inhibition of lmmediate and delayed type hypersensitivity by the $70 \%$ ethanolic extract from Fructus Kochiae Scopariae," Chinese Journal of Modern Applied Pharmacy, vol. 18, 2001.

[21] Z. Xiao, S. Xiao, Y. Zhang, T. Pan, and B. Ouyang, "The antiinflammatory effect of fructus kochiae on allergic contact dermatitis rats via pERK1/2/TLR4/NF- $\kappa \mathrm{B}$ pathway activation," Evidence-Based Complementary and Alternative Medicine, vol. 4, Article ID 1096920, 2018.

[22] S. Jo, J. Ryu, H.-Y. Han, G. Lee, M. H. Ryu, and H. Kim, "Antiinflammatory activity of Kochia scoparia fruit on contact dermatitis in mice," Molecular Medicine Reports, vol. 13, no. 2, pp. 1695-1700, 2016.

[23] D. Yue, X. Yufeng, and L. Silong, "Studies on the hypoglycemic mechanism of n-butanol fraction from Fructus Kochiae (地肤子)," Pharmacology and Clinics of Chinese Materia Medica, vol. 19, 2003.

[24] D. Yue, X. Yufeng, and Y. Li, "Effect of n-butanol fraction from Fructus Kochiae(地肤子) on intestinal motility," Pharmacology $\backslash$ se $\mid$ sclinics of Chinese Materia Medica, vol. 20, 2004.

[25] H. Matsuda, Y. Li, T. Murakami, N. Matsumura, J. Yamahara, and M. Yoshikawa, "Antidiabetic principles of natural medicines. III. Structure-related inhibitory activity and action mode of oleanolic acid glycosides on hypoglycemic activity," Chemical \& Pharmaceutical Bulletin, vol. 46, no. 9, pp. 1399-1403, 1998.

[26] J. Wang, L. Yuan, H. Xiao, C. Xiao, Y. Wang, and X. Liu, "Momordin Ic induces HepG2 cell apoptosis through MAPK and PI3K/Akt-mediated mitochondrial pathways," Apoptosis, vol. 18, no. 6, pp. 751-765, 2013.

[27] Y. Mi, C. Xiao, Q. Du, W. Wu, G. Qi, and X. Liu, "Momordin Ic couples apoptosis with autophagy in human hepatoblastoma cancer cells by reactive oxygen species (ROS)-mediated PI3K/Akt and MAPK signaling pathways," Free Radical Biology and Medicine, vol. 90, pp. 230-242, 2016.

[28] J. Wang, L. Yuan, H. Xiao et al., "A novel mechanism for momordin Ic-induced HepG2 apoptosis: involvement of PI3K- and MAPK-dependent PPAR $\gamma$ activation," Food \& Function, vol. 5, no. 5, pp. 859-868, 2014.

[29] J. Wang, Y. Han, M. Wang, Q. Zhao, X. Chen, and X. Liu, "Natural triterpenoid saponin Momordin Ic suppresses HepG2 cell invasion via COX-2 inhibition and PPAR $\gamma$ activation," Toxicol In Vitro, vol. 65, Article ID 104784, 2020.

[30] J. Wang, Q. Liu, H. Xiao, X. Luo, and X. Liu, "Suppressive effects of Momordin Ic on HepG2 cell migration and invasion by regulating MMP-9 and adhesion molecules: involvement of p38 and JNK pathways," Toxicology in Vitro, vol. 56, pp. 75-83, 2019.

[31] H.-D. Cho, J.-H. Kim, J.-K. Park, S.-M. Hong, D.-H. Kim, and K.-I. Seo, "Kochia scoparia seed extract suppresses VEGFinduced angiogenesis via modulating VEGF receptor 2 and PI3K/AKT/mTOR pathways," Pharmaceutical Biology, vol. 57, no. 1, pp. 684-693, 2019.

[32] J. Wu, H. Lei, J. Zhang et al., "Momordin Ic, a new natural SENP1 inhibitor, inhibits prostate cancer cell proliferation," Oncotarget, vol. 7, no. 37, pp. 58995-59005, 2016.

[33] J. N. Wang, Q. Y. Liu, C. H. Yao et al., "[Normative research on bacteriostasis and relieving itching external therapeutic function of kochiae fructus]," Zhong Yao Cai, vol. 35, no. 12, pp. 1974-1977, 2012.

[34] J. Wu, S. Guang-Lub, S. U. Xue-Youa, W. Zhe-Yia, and W. You-Niana, A Preliminary Study On Bioactivity Of Extracts From Fructus Kochiae Against Several Phytopathogens, Journal of Beijing University of Agriculture, Beijing, China, 2008.

[35] Y. Wang and C. Sui, "Pharmacology and toxicity comparation of difuzi and Li," Journal of Modern Applied Pharmacy, vol. 12, no. 4, pp. 10-12, 1995.

[36] M. Kubo, H. Matsuda, Y. Dai, Y. Ido, and M. Yoshikawa, "[Studies on Kochiae Fructus. I. Antipruritogenic effect of 
$70 \%$ ethanol extract from kochiae fructus and its active component]," Yakugaku Zasshi, vol. 117, no. 4, pp. 193-201, 1997.

[37] D. Le, Z. Jiarong, and B. Yonghong, “The regulatory effect of fructus kochiae on Th cell activity in rats with dilated cardiomyopathy," Jiangsu Medical Journal, vol. 38, 2012.

[38] N.-Y. Kim, M.-K. Lee, M.-J. Park et al., "Momordin Ic and oleanolic acid from Kochiae Fructus reduce carbon tetrachloride-induced hepatotoxicity in rats," Journal of Medicinal Food, vol. 8, no. 2, pp. 177-183, 2005.

[39] H. Matsuda, Y. Li, T. Murakami, J. Yamahara, and M. Yoshikawa, "Protective effects of oleanolic acid oligoglycosides on ethanol- or indomethacin-induced gastric mucosal lesions in rats," Life Sciences, vol. 63, no. 17, pp. PL245-PL250, 1998.

[40] Y. H. Hwang, J. W. Lee, E.-R. Hahm et al., "Momordin I, an inhibitor of AP-1, suppressed osteoclastogenesis through inhibition of NF- $\kappa \mathrm{B}$ and AP-1 and also reduced osteoclast activity and survival," Biochemical and Biophysical Research Communications, vol. 337, no. 3, pp. 815-823, 2005.

[41] M. Yan, W. Zhang, G. Luo, S. Demetra, X. Wang, and C. Chen, "Screening of antibacterial active constituents from fructus kochiae," Chemistry \& Bioengineering, vol. 36, no. 2, pp. 28-31, 2019.

[42] Z. Xiao, S. Xiao, Y. Zhang, T. Pan, and B. Ouyang, "The antiinflammatory effect of fructus kochiae on allergic contact dermatitis rats via $\mathrm{pERK} 1 / 2 / \mathrm{TLR} 4 / \mathrm{NF}-\mathrm{kappaB}$ pathway activation," Evidence-Based Complementary and Alternative Medicine, vol. 2018, Article ID 1096920, 12 pages, 2018.

[43] T. Bawa-Khalfe, J. Cheng, Z. Wang, and E. T. H. Yeh, "Induction of the SUMO-specific protease 1 transcription by the androgen receptor in prostate cancer cells," Journal of Biological Chemistry, vol. 282, no. 52, pp. 37341-37349, 2007.

[44] J. Cheng, X. Kang, S. Zhang, and E. T. H. Yeh, "SUMOspecific protease 1 is essential for stabilization of HIF $1 \alpha$ during hypoxia," Cell, vol. 131, no. 3, pp. 584-595, 2007.

[45] T. Bawa-Khalfe, J. Cheng, S.-H. Lin, M. M. Ittmann, and E. T. H. Yeh, "SENP1 induces prostatic intraepithelial neoplasia through multiple mechanisms," Journal of Biological Chemistry, vol. 285, no. 33, pp. 25859-25866, 2010.

[46] Q. Wang, N. Xia, T. Li et al., "SUMO-specific protease 1 promotes prostate cancer progression and metastasis," Oncogene, vol. 32, no. 19, pp. 2493-2498, 2013.

[47] L. I. Pei-Yuan, L. N. Huo, S. U. Wei, L. Y. Deng, M. P. Peng, and Z. X. Zhang, Radical Scavenging Activity And Total Phenolics Content Of Kochia Scoparia, Hubei Agricultural Sciences, Wuhan, China, 2016.

[48] J. Wang, L. Hou, H. Zhang, Y. Zhang, and X. F. Chen, Study On Comparison Of Free Radical Scavenging Activities In The Ethanol Kochia Scoparia Extract, Journal of Shaanxi University of Science \& Technology, Xi'an, China, 2017.

[49] Z. Hao, "Antioxidant activities of flavonoids extraction from Kochia scoparia," Chemical Industry Times, 2012.

[50] M. Yan, W. Zhang, G. Luo, X. Wang, S. Feng, and N. Zhao, "Screening chemical constituents with antioxidative activity from the kochiae fructus," Chinese Medicine Journal of Research \& Practice, vol. 33, no. 4, pp. 29-32, 2019.

[51] H. Jeon, D. H. Kim, Y. H. Nho, J. E. Park, S. N. Kim, and E. H. Choi, "A mixture of extracts of Kochia scoparia and rosa multiflora with PPAR $\alpha / \gamma$ dual agonistic effects prevents photoaging in hairless mice," International Journal of Molecular Sciences, vol. 17, no. 11, 2016.

[52] Y. F. Xia, Q. Wang, X. U. De Ran, H. Wang, and Y. E. Wen Cai, "Determination of the content of momordin Ic in Kochia scoparia by HPLC," Journal of China Pharmaceutical University, vol. 33, no. 3, pp. 216-218, 2002.

[53] X. Y. Feng, W. Qiang, and D. Yue, "Changes of saponin content in Kochia scoparia fruit in different collecting times," Journal of Plant Resources \& Environment, vol. 11, 2002.

[54] Y. F. Xia, W. Qiang, D. Yue, and P. Kai, "Determination of the content of saponin in Kochia scoparia fruits from different producing areas," China Journal of Chinese Materia Medica, vol. 27, no. 12, pp. 890-893, 2002.

[55] X. Zuoqi, W. Xiaoke, O. Bo et al., "Simultaneous determination of rutin and quercetin in kochiae fructus by hplc," China Medical Herald, vol. 13, no. 21, pp. 146-152, 2016.

[56] Z. Q. Xiao, B. Ouyang, T. Pan, and X. K. Wen, "Study on HPLC fingerprint of kochiae fructus and their cluster analysis," Guiding Journal of Traditional Chinese Medicine \& Pharmacy, vol. 23, 2017. 